\title{
Unveiling early black hole growth with multifrequency gravitational wave observations
}

\author{
Rosa Valiante ${ }^{\circledR}, 1,2 \star$ Monica Colpi, ${ }^{3,4}$ Raffaella Schneider ${ }^{\circledR}, 1,2,5$ Alberto Mangiagli, ${ }^{3,4}$ Matteo Bonetti ${ }^{\circledR}, 3$ \\ Giulia Cerini, ${ }^{6}$ Stephen Fairhurst, ${ }^{7}$ Francesco Haardt, ${ }^{4,8}$ Cameron Mills $^{7}$ and Alberto Sesana ${ }^{3}$ \\ ${ }^{1}$ INAF - Osservatorio Astronomico di Roma, via di Frascati 33, I-00078 Monteporzio Catone, Italy \\ ${ }^{2}$ INFN, Sezione di Roma I, P.le Aldo Moro 2, I-00185 Roma, Italy \\ ${ }^{3}$ Department of Physics, University of Milano-Bicocca, Piazza della Scienza 3, I-20126 Milano, Italy \\ ${ }^{4}$ National Institute of Nuclear Physics INFN, Milano-Bicocca, Piazza della Scienza 3, I-20126 Milano, Italy \\ ${ }^{5}$ Dipartimento di Fisica, Universitá di Roma 'La Sapienza', P.le Aldo Moro 2, I-00185 Roma, Italy \\ ${ }^{6}$ Department of Physics, University of Miami, 1320 Campo Sano Drive, Coral Gables, FL 33124, USA \\ ${ }^{7}$ Gravity Exploration Institute, School of Physics and Astronomy, Cardiff University, Cardiff CF24 3AA, UK \\ ${ }^{8}$ DiSAT, Universitá dell'Insubria, via Valleggio 11, I-22100 Como, Italy
}

Accepted 2020 October 27. Received 2020 October 12; in original form 2020 July 10

\begin{abstract}
Third-generation ground-based gravitational wave interferometers, like the Einstein Telescope (ET), Cosmic Explorer, and the Laser Interferometer Space Antenna (LISA), will detect coalescing binary black holes over a wide mass spectrum and across all cosmic epochs. We track the cosmological growth of the earliest light and heavy seeds that swiftly transit into the supermassive domain using a semi-analytical model for the formation of quasars at $z=6.4,2$, and 0.2 , in which we follow black hole coalescences driven by triple interactions. We find that light-seed binaries of several $10^{2} \mathrm{M}_{\odot}$ are accessible to ET with a signal-to-noise ratio $(\mathrm{S} / \mathrm{N})$ of $10-20$ at $6<z<15$. They then enter the LISA domain with larger S/N as they grow to a few $10^{4} \mathrm{M}_{\odot}$. Detecting their gravitational signal would provide first time evidence that light seeds form, grow, and dynamically pair during galaxy mergers. The electromagnetic emission of accreting black holes of similar mass and redshift is too faint to be detected even for the deepest future facilities. ET will be our only chance to discover light seeds forming at cosmic dawn. At $2<z<8$, we predict a population of 'starved binaries', long-lived marginally growing light-seed pairs, to be loud sources in the ET bandwidth $(\mathrm{S} / \mathrm{N}>20)$. Mergers involving heavy seeds $\left(\sim 10^{5}-10^{6} \mathrm{M}_{\odot}\right)$ would be within reach up to $z=20$ in the LISA frequency domain. The lower $z$ model predicts 11.25 (18.7) ET (LISA) events per year, overall.
\end{abstract}

Key words: galaxies: evolution-galaxies: high-redshift-quasars: supermassive black holes - black hole mergers.

\section{INTRODUCTION}

The discovery of luminous quasars powered by accretion on to $10^{9}-10^{10} \mathrm{M}_{\odot}$ supermassive black holes (SMBHs) at redshift as early as $z \sim 7.5$ (Bañados et al. 2018; Yang et al. 2020), only $\sim 800 \mathrm{Myr}$ after the big bang, has revolutionized our view on how these giants formed before the epoch of cosmic reionization (Bañados et al. 2016). They represent the tip of an underlying population of much fainter active galactic nuclei (AGNs; Matsuoka et al. 2018) that are the least known in terms of basic demographics, birth, and growth. As gas is likely the primary fuel for their growth (Marconi et al. 2004; Merloni, Rudnick \& Di Matteo 2004; Kormendy \& Ho 2013; Trakhtenbrot 2020), this observation hints to the existence, at redshifts $z>7$, of a population of seed black holes (BHs) of yet unconstrained initial mass, in the range from about $\sim 100$ to $\sim 10^{5} \mathrm{M}_{\odot}$ from which the giants have grown. This interval is often referred to as intermediate (between stellar-mass BHs and SMBHs), with light seeds in the range

*E-mail: rosa.valiante@inaf.it between $\sim 10^{2} \mathrm{M}_{\odot}$ and a few $10^{3} \mathrm{M}_{\odot}$, and heavy seeds in the range between $10^{4}$ and $10^{6} \mathrm{M}_{\odot}$ as extremes (Valiante et al. 2017).

The origin of seeds is not known yet, nor the mechanisms leading to their swift evolution to become high- $z$ quasars (Volonteri 2010; Schleicher et al. 2013; Johnson \& Haardt 2016; Latif \& Ferrara 2016; Inayoshi, Visbal \& Haiman 2019). Various avenues of formation have been proposed:

Light seeds: massive stars collapsing into stellar BHs beyond the pair instability gap (Heger \& Woosley 2010), with masses of a few $10^{2} \mathrm{M}_{\odot}$ forming in metal-free/poor dark matter (DM) haloes at redshifts $z$ as large as $\sim 20-30$ (Madau \& Rees 2001; Abel, Bryan \& Norman 2002; Heger et al. 2003; Yoshida, Omukai \& Hernquist 2008; Hirano et al. 2014, 2015).

Medium-weight seeds: very massive stars, resulting from runaway stellar mergers, in compact star clusters forming at $z \sim 10$ (Devecchi et al. 2012; Mapelli 2016; Reinoso et al. 2018). Here, stellar masses of $\sim 200-10^{3} \mathrm{M}_{\odot}$ are not set by the fragmentation properties of the birth gas clouds but by stellar collisions ruled by the dynamics inside the earliest dense nuclear star clusters. Alternatively, they may form in runway gravitational wave (GW)driven coalescences of stellar BHs in star clusters subject to major 
gas inflows, at the centre of pre-galactic discs forming at $z \sim 10$ (Davies, Miller \& Bellovary 2011; Lupi et al. 2014);

Heavy seeds: supermassive (proto)-stars of $\sim 10^{4-6} \mathrm{M}_{\odot}$ growing through continued and fast accretion within their birth clouds, collapsing directly on to a $\mathrm{BH}$, the so-called direct-collapse $\mathrm{BH}$ $(\mathrm{DCBH})$ scenario, driven by general relativistic instabilities or fuel exhaustion (Bromm \& Loeb 2004; Begelman, Volonteri \& Rees 2006; Inayoshi \& Omukai 2012; Inayoshi, Omukai \& Tasker 2014; Umeda et al. 2016). These are considered to be rare seeds due to their contrived birth environmental conditions (Agarwal et al. 2012; Latif et al. 2013; Dijkstra, Ferrara \& Mesinger 2014; Chon et al. 2016; Habouzit et al. 2016; Valiante et al. 2016; Regan et al. 2017). Intense UV radiation from adjacent star-forming regions and large infall rates of metal-free/poor gas are required to suppress fragmentation of the birth cloud and to feed the central proto-star. Even in slightly enriched haloes $\left(Z<10^{-3} Z_{\odot}\right)$, where fragmentation takes place, infalling, metal-poor, material preferentially feeds the primary protostar (the first to form in the cloud) that grows supermassive (the so-called supercompetitive accretion scenario; Chon \& Omukai 2020). Alternatively, the formation of heavy seeds may be aided by dynamical heating during rapid mass growth of low-mass haloes in overdense regions at high redshifts (Wise et al. 2019) or by massive nuclear inflows in major gas-rich galaxy mergers at lower redshift (Mayer et al. 2015).

Currently, the only way to infer information on BHs of $\sim 10^{5} \mathrm{M}_{\odot}$ is by looking at local dwarf galaxies (Baldassare et al. 2015; Reines \& Volonteri 2015; Mezcua et al. 2016, 2018) where observational signatures of seed formation are expected to be strong (Habouzit et al. 2016). Although the faint-end tail of the $z \sim 6$ AGN luminosity function has been sampled down to absolute magnitude of $M_{1459}$ $=-22$ mag (Matsuoka et al. 2018), no observational signatures of fainter AGN, possibly powered by $\mathrm{BHs}$ of $<10^{7} \mathrm{M}_{\odot}$, have been found at higher redshifts. The non-detection of faint high- $z$ AGNs may be a consequence of their low active fraction $(\sim 0.1$ per cent at $z>7$; Pezzulli et al. 2017a) and/or of their relatively low number density (Habouzit et al. 2016; Valiante et al. 2016; Cowie et al. 2020, but see Wise et al. 2019).

In the next decades, with the advent of the foremost electromagnetic (EM) facilities and of the next generation of ground- and space-based GW interferometers, breakthrough in this field will be accomplished exploiting jointly the power of traditional Astrophysics with the nascent multifrequency GW Astronomy.

Light waves on the one side: The Square Kilometer Array in radio, the James Webb Space Telescope (JWST) and the Extremely Large Telescope in the optical and near-infrared, the Advanced Telescopes for High Energy Astrophysics Athena, and the mission-concept Lynx in the X-rays will provide new information on the earliest accreting BHs, the dimmest AGN of the low-mass tail of SMBH population, and binary or/and multiple AGNs in interacting systems, and ultimately will let us identify the EM counterparts of the loudest GW signals from merging massive BHs (Dal Canton et al. 2019; McGee, Sesana \& Vecchio 2020).

$G W s$ on the other side: Third-generation ground-based interferometers such as Einstein Telescope (ET; Punturo et al. 2010; Sathyaprakash et al. 2012) and Cosmic Explorer (CE; Abbott et al. 2017; Reitze et al. 2019) will capture the GW signal from millions of coalescing stellar binary BHs $(\mathrm{BBHs})$ detectable out to $z \sim 10-15$. In particular, ET, with a higher sensitivity at the lowest frequencies around $3-10 \mathrm{~Hz}$, has the potential of discovering mergers of BBHs with masses of up to a few $100 \mathrm{M}_{\odot}$, characteristic of the earliest stellar and seed $\mathrm{BH}$ populations and $\mathrm{BBH}$ s of a few $10^{3} \mathrm{M}_{\odot}$ at moderate redshifts (Kalogera et al. 2019; Maggiore et al. 2019). Space-based interferometers such as the Laser Interferometer Space Antenna (LISA), the interferometer TianQin under design (Luo et al. 2016), and the proposed Taiji program (Ruan et al. 2018) will instead detect the GW signals from massive BBH coalescences (from $\sim 10^{4} \mathrm{M}_{\odot}$ up to about $\sim 10^{7} \mathrm{M}_{\odot}$ ) across all cosmic ages providing the first ever census of this new population of BHs that formed in the aftermath of galaxy collisions (Amaro-Seoane et al. 2017; Colpi et al. 2019). Thus, future GW observatories together will detect the signal emitted by coalescing BBHs over a wide mass spectrum, from the stellar to the massive, through the formation of seeds, and across all cosmic epochs.

Seeds are expected to grow via accretion of surrounding gas in primeval DM haloes. Their growth might be Eddington limited leading to an $e$-fold increase in the mass on time-scales of a few $100 \mathrm{Myr}$ if uninterrupted. Growth may occur at super-Eddington rates if seeds are surrounded by radiatively inefficient slim discs (Madau, Haardt \& Dotti 2014; Volonteri, Silk \& Dubus 2015; Pezzulli et al. $2017 \mathrm{~b}$ ) or at supra-exponential rates if embedded in star clusters fed by dense cold gas, expected to be ubiquitous in the highredshift Universe (Alexander \& Natarajan 2014). However, BHs invariably participate in the assembly of cosmic structures during their evolution, possibly growing also through coalescences, in addition to gas accretion (Volonteri, Haardt \& Madau 2003; Sesana, Volonteri \& Haardt 2007; Valiante et al. 2016). This implies that seed BHs might pair and merge shortly after their formation in the earliest halo-halo merger events, becoming high- $z$, sources of GWs at frequencies of $\sim 3-10 \mathrm{~Hz}$, in the ET frequency band (light seeds), and/or $100 \mu \mathrm{Hz}-100 \mathrm{mHz}$, the LISA domain (medium-weight and heavy seeds).

In this paper, we aim at exploring the emergence of cosmologically driven pairs of seed BHs merging in the aftermath of halo-halo collisions, following their growth via accretion and mergers to track their swift transit across the ET and LISA bandwidths, as GW sources. To this purpose, we improve upon GAMETE/QSODUST (GQD), the Semi-Analytical Model (SAM) presented in Valiante et al. (2016, 2018a). Developed to model the formation and evolution of high- $z$ quasars, GQD includes a refined seeding prescription for both light and heavy seeds combing chemical and radiative properties of the environment in haloes selected among $z>10$ progenitors of $z>$ 6 quasars.

In addition, in Valiante et al. (2018a, b) we followed the early growth of a seed via gas accretion only inside an evolving unperturbed halo, before the information on its birth environment (and hence on the nature of the $\mathrm{BH}$ seed) was erased as a consequence of a halo-halo merger. By processing the radiation emitted by the stars and accreting BHs through gas and dust, we showed that the most massive $\left(>10^{6} \mathrm{M}_{\odot}\right.$ ) and rapidly growing seeds would be easily detected by future (EM) missions, like Athena and JWST (Pacucci et al. 2015; Natarajan et al. 2017), up to $z \sim 15$ (Valiante et al. 2018b). By contrast, lighter accreting BHs with a mass $\lesssim 10^{3} \mathrm{M}_{\odot}$ would remain undetectable due to their weaker emission, showing the limiting power of EM observations in detecting seed BHs. In this paper, we aim at exploring whether future GW telescopes would allow us to discover in a unique way the formation and evolution of the earliest seeds and their potential link with SMBHs (Colpi 2019).

Using GQD, we focus here on the histories of three DM haloes, of equal mass, each hosting a quasar shining at a different redshift: $z_{\mathrm{QSO}}$ $\sim 6.4$, near the epoch of reionization of the intergalactic hydrogen, at $z_{\mathrm{QSO}} \sim 2$, near the peak of the cosmic star formation rate (SFR) density in the Universe, and at $z_{\mathrm{QSO}}=0.2$, during the fading of the AGN activity and quenching of the SFR. We follow the hierarchical formation pathways of these quasars by describing seed growth ruled 
by accretion episodes and mergers in multiple DM halo collisions, including in GQD a prescription to track their dynamics down to coalescence, driven by triple BH interactions (Bonetti et al. 2016, 2018a).

The paper is organized as follows. The semi-analytical approach is summarized in Section 2, while in Section 3 the new features of the model are described. In Sections 4 and 5, the emergence of BBHs within our model is analysed in view of the future GW and EM facilities. A critical discussion of our approach is presented in Section 6. Finally, our main conclusions are drawn in Section 7.

\section{THE QUASAR EVOLUTION MODEL}

In this section, we summarize the main features of our dataconstrained SAM, GQD, and refer the interested reader to Valiante et al. (2016, 2018a, b), and references therein, for details. The model follows the formation and evolution of individual quasars, powered by accretion on to SMBHs, and their host galaxies, observed at high redshift, with particular attention to $z>6$ systems, like SDSS $\mathrm{J} 1148+5251(\mathrm{~J} 1148)$ at $z_{\mathrm{QSO}}=6.4$ (Valiante et al. 2011, 2016). GQD has been extensively tested against a sample of $z_{\mathrm{QSO}}>5$ quasars, well reproducing their observed properties (Valiante et al. 2014). For the purposes of this work, we extend the analysis to lower redshift analogues, i.e. quasars at $z_{\mathrm{QSO}}=2$ and 0.2 , respectively. The evolution of each DM halo is described using semi-analytically reconstructed merger histories.

\subsection{Dark matter halo}

With GQD, we produce for each simulated quasar 10 merger tree realizations of a DM halo of $M_{0}=10^{13} \mathrm{M}_{\odot}$, in which the luminous quasar is expected to reside. ${ }^{1}$ This DM halo is decomposed into progressively less massive fragments, called progenitors, through a binary Monte Carlo algorithm with mass accretion based on the Extended Press-Schechter (PS) formalism (Press \& Schechter 1974).

At a given redshift $z$ along the merger tree, the minimum mass of a resolved structure (virialized progenitor), i.e. the merger tree mass resolution, is described as

$M_{\mathrm{res}}(z)=10^{-3} M_{0}\left(\frac{1+z}{1+z_{\mathrm{QSO}}}\right)^{\beta}$,

where $M_{0}=10^{13} \mathrm{M}_{\odot}$ is the same for the three quasars and the parameter $\beta$ is assumed to be $-7.5,-4.3$, and -3.0 for $z_{\mathrm{QSO}}=6.4$, 2 , and 0.2 , respectively (Valiante et al. 2016), so that at $z=24(z=$ $\left.z_{\mathrm{QSO}}\right) M_{\mathrm{res}} \sim 10^{6}\left(10^{10}\right) \mathrm{M}_{\odot}$. Non-resolved structures with $M<M_{\text {res }}$ account for the external, intergalactic medium (IGM) from which progenitor haloes accrete mass.

The characteristic redshift interval of the merger tree models, $\Delta z$, the functional form of the mass resolution, and the value of the parameter $\beta$ have been chosen to (i) resolve mini-haloes (i.e. those DM progenitors with virial temperatures in the range $1200 \mathrm{~K}$ $\leq T_{\text {vir }}<10^{4} \mathrm{~K}$ ) at high redshift, (ii) prevent the formation of multiple fragments ( $>2$ per progenitor halo, as required by the binary algorithm), (iii) reproduce the Extended PS halo mass functions, and (iv) limit the computational times. These requirements determine the redshift distribution and total number of progenitors forming between $z=24$ and $z_{\mathrm{QSO}}$, which is higher for lower $z_{\mathrm{QSO}}$ simulations.

\footnotetext{
${ }^{1}$ It is commonly believed that $\left[10^{12}-10^{13}\right] \mathrm{M}_{\odot}$ host DM haloes are required to match the observed space density of $z \sim 6$ quasars (Fan et al. 2004 and see Valiante et al. 2011 for a discussion).
}

According to equation (2.1) mini-haloes of $\sim 10^{6}-10^{8} \mathrm{M}_{\odot}$ are resolved at $z>13,8$, and 5 in the merger trees of the $z_{\mathrm{QS} 0}=6.4,2$, and 0.2 simulated quasar hosts, respectively. These low-mass haloes are expected to be the first formation sites of Population III stars, at $z \sim 20-30$, and of light seeds. Along each reconstructed merger tree, GQD consistently follows the evolution of each progenitor galaxy and its nuclear $\mathrm{BH}$, running forward in time from $z=24$ to $z_{\mathrm{Q}} \mathrm{SO}$.

The adopted resolution mass does not have a significant impact on the analysis presented here since, close to the final redshift, accretion and merging of low-mass haloes increase their mass above the resolution. Furthermore, chemical and radiative feedbacks inhibit the formation of $\mathrm{BH}$ seeds when $z<17(13,12)$ for the quasar models with $z_{\mathrm{QSO}}=6.4(2,0.2)($ see Section 4.1$)$.

\subsection{Quasar's progenitor galaxies}

The (co-)evolution of BHs and their host galaxies is a complex process, regulated by the interplay between chemical, mechanical, and radiative feedbacks. In the framework of mainstream structure formation scenarios, seeds grow by accreting at a rate regulated by the reservoir of dense, cold gas present in their neighbourhood. This, in turn, is set by the baryon cycle of the forming host galaxy that gains mass through gas inflows from the external IGM, consumes mass to fuel star formation, and loses mass via winds powered by supernova explosions and by the radiation that the BH feeds back into the interstellar medium (ISM).

Mass exchanges with the IGM, genetic (in-situ) ISM metal enrichment of the galaxies, and the intensity of the permeating UV field all contribute to determine the efficiency of star formation (especially in mini-haloes), the duration of the Pop III star forming epoch, and the number and nature of $\mathrm{BH}$ seeds that form.

\subsubsection{Starformation}

In each progenitor galaxy, we convert gas into stars at a rate that is given by

$\mathrm{SFR}=f_{\mathrm{cool}} M_{\mathrm{gas}} \epsilon / t_{\mathrm{dyn}}(z)$,

where SFR is the star formation rate and $t_{\mathrm{dyn}}(z)=R_{\mathrm{vir}} / v_{\mathrm{e}}$ is the redshift-dependent dynamical time-scale $\left(R_{\text {vir }}\right.$ and $v_{\mathrm{e}}$ being the halo virial radius and escape velocity, respectively). In our model, stars form through a series of quiescent $\left(\epsilon=\epsilon_{\text {quiesc }}\right)$ and major-merger enhanced bursts $\left(\epsilon=\epsilon_{\text {quiesc }}+\epsilon_{\text {burst }}\right)$. The quiescent star formation efficiency is a free parameter of the model and the choice of its value is discussed in Section 4. The parameter $\epsilon_{\text {burst }}$ accounts for the efficiency enhancement due to major galaxy mergers, that is the coalescences of two DM haloes with mass ratios $\mu_{\mathrm{DM}}>1 / 4$ (least massive over most massive). In GQD $\epsilon_{\text {burst }}$ is a function of $\mu_{\mathrm{DM}}$, computed as a Gaussian distribution with $\sigma_{\text {burst }}=0.05$ (we have $\epsilon_{\text {burst }}=8$ for $\mu_{\mathrm{DM}}=1 / 4$; see Valiante et al. 2011).

Finally, the quantity $f_{\text {cool }}$ is the ratio between the total mass of gas enclosed in the halo virial radius and the gas mass within the "cooling radius' $r_{\text {cool }}$, the radius at which the cooling time, $t_{\text {cool }}$, equals the freefall time, $t_{\mathrm{ff}}$. The value of $f_{\text {cool }}$ represents the reduced star formation efficiency of mini-haloes $\left(f_{\text {cool }}<1\right)$ with respect to atomic cooling haloes $\left(T_{\text {vir }} \geq 10^{4} \mathrm{~K}, f_{\text {cool }}=1\right)$, as described in Valiante et al. (2016) and de Bennassuti et al. (2017). In mini-haloes, in fact, the fraction of the available gas that can cool and form stars strongly depends on halo properties (virial temperature, redshift, and gas metallicity) and on the intensity of illuminating far-UV radiation, which can photodissociate $\mathrm{H}_{2}$ molecules, the main coolant in these haloes. 
For each stellar population formed via equation (2), we adopt a Larson initial mass function (IMF; Larson 1998) to describe the stellar mass spectrum. The first generation of stars (Pop III stars) forms in pristine/metal-poor galaxies with a metallicity $Z<Z_{\mathrm{cr}} \sim$ $10^{-3.8} Z_{\odot}$ (Schneider et al. 2002, 2003, 2012) and is characterized by a 'top-heavy' IMF with masses in the range [10-300 $\mathrm{M}_{\odot}$ ] and a characteristic mass of $m_{\mathrm{ch}}=20 \mathrm{M}_{\odot}$. Conversely, Pop II stars form out of chemically enriched gas $\left(Z>Z_{\mathrm{cr}}\right)$ following a standard, Salpeterlike, IMF (approximated by a Larson IMF with a characteristic mass of $\left.m_{\mathrm{ch}}=0.35 \mathrm{M}_{\odot}\right)$ in the mass range [0.1-100 $\left.\mathrm{M}_{\odot}\right]$.

In low-efficiency starburst, when the total stellar mass formed in $M_{\text {star }}<10^{6} \mathrm{M}_{\odot}$, the intrinsic top-heavy Pop III stellar IMF is stochastically sampled, randomly extracting single stars from the [10-300 $\mathrm{M}_{\odot}$ ] mass range until the cumulative value of $M_{\text {star }}$ is reached.

\subsection{Black hole seeds}

Following Valiante et al. (2016), BH seeds form under conditions set by the efficiency of metal and dust enrichment and by the intensity of the far-UV radiation.

Depending on the random sampling of the IMF described above, light seeds form in both mini-haloes and atomic cooling haloes by the collapse of [40-140 $\mathrm{M}_{\odot}$ ] and [260-300 $\mathrm{M}_{\odot}$ ] Pop III stars (consistent with the existence of a pair instability mass gap). The resulting BHs (i.e. the collapsed remnants) are as massive as their progenitors, assuming non-rotating primordial stars, for which no mass-loss is expected (Heger \& Woosley 2002). Only the most massive BH of each population is assumed to settle in the galaxy centre.

In our seeding prescription, heavy $\mathrm{BH}$ seeds of $10^{5} \mathrm{M}_{\odot}$ form in metal-poor $\left(Z<Z_{\text {cr }}\right)$, atomic cooling haloes, when the cumulative Lyman Werner (LW) emission (from stars and accreting BHs in all galaxies), $J_{\mathrm{LW}}$, becomes larger than a critical threshold $J_{\mathrm{cr}} \equiv$ $300 \times 10^{-21} \mathrm{erg} \mathrm{s}^{-1} \mathrm{~Hz}^{-1} \mathrm{~cm}^{-1} \mathrm{sr}^{-1}$ (for a discussion, see Valiante et al. 2017, and references therein).

The subsequent growth of nuclear BHs is driven by accretion of gas and mergers with other BHs. To describe the gas accretion rate, we adopt the Bondi-Hoyle-Lyttleton formula, re-scaled by a factor $\alpha_{\mathrm{BH}}$ that accounts for the higher central densities around $\mathrm{BHs}$, as required by sub-grid prescriptions adopted in SAM and large-volume numerical simulations (e.g. Di Matteo, Springel \& Hernquist 2005; Booth \& Schaye 2009). In addition, we assume that the computed $\mathrm{BH}$ accretion rate cannot exceed the Eddington limit (see Valiante et al. 2014, for details).

\subsection{Stellar and black hole feedback}

After each star formation episode, the galaxies ISM is polluted with metals and dust produced by supernovae (end products of mainsequence stars of 10-40 $\mathrm{M}_{\odot}$ ) and asymptotic giant branch stars (with an initial mass of $1-8 \mathrm{M}_{\odot}$ ). The injection of fresh metals and dust produced by stars is regulated by the stellar lifetimes and depends on the initial mass and metallicity of the stars. We follow dust cycling in the two-phase ISM by accounting for SN shock destruction in the hot, diffuse medium and grain growth in cold, dense molecular clouds (see Valiante et al. 2014; de Bennassuti et al. 2014, for details). The stellar products can then be ejected out of the ISM, on scales larger than the halo virial radius. The energy released by star formation and $\mathrm{BH}$ accretion couples with the gas, heating and accelerating it.

We describe mechanical feedback by means of energy-driven winds: Galaxy-scale gas outflows are launched from the galaxy polluting the IGM with metals and dust. In our models, we assume that a fixed fraction of the energy deposited by $\mathrm{SN}$ explosions and $\mathrm{BH}$ accretion, $\epsilon_{\mathrm{w}, \mathrm{SN}}=2 \times 10^{-3}$ and $\epsilon_{\mathrm{w}, \mathrm{AGN}}=2.5 \times 10^{-3}$, respectively, drives the massive gas outflows (see Valiante et al. 2016, for details).

We compute the time-dependent cumulative LW radiation, $J_{\mathrm{LW}}$, coming from all the emitting sources, stars, and AGNs (Valiante et al. 2016). At each redshift, this can be considered as the background radiation permeating a comoving volume ${ }^{2}$ of $50 \mathrm{Mpc}^{3}$ (see discussion in Valiante et al. 2017, 2018b).

\section{THE DYNAMICS OF BINARY BLACK HOLES}

A description of $\mathrm{BH}$ dynamics in cosmological frameworks has been included, with different approaches, in several semi-analytical models so far (e.g. Volonteri et al. 2003; Barausse, Morozova \& Rezzolla 2012; Klein et al. 2016; Bonetti et al. 2019; Katz et al. 2020), and, recently, in few large-scales simulations (associating time delays to BBHs in post-processing; see e.g. Kelley, Blecha \& Hernquist 2017; Volonteri et al. 2020).

In our previous models, we assumed that during major mergers ${ }^{3}$ the BHs coalesce instantaneously as their hosts merge. In particular, in Valiante et al. (2016) BHs coalesce right away, over the merger tree time interval (that is typically of a few Myr), while in minor mergers the most massive $\mathrm{BH}$ remains in the centre of the newly formed galaxy and the less massive is considered as a satellite and its evolution is no longer followed.

However, BH coalescences occur with a time delay compared to the typical time of the galaxy merger (Colpi 2014). GW emission drives the inspiral on time-scales of less than $\lesssim$ Gyr only when the two $\mathrm{BHs}$ reach relative separations of milliparsec or smaller, depending on the binary mass, mass ratio, and orbital eccentricity.

During halo mergers, the two nuclear $\mathrm{BHs}$ can be driven to such minuscule galactic distances by DM/stellar and gas dynamical torques that control their sinking from the kpc scale downward. Hence, the formation of BBHs in halo mergers and their hardening on time-scales shorter than the cosmic time are an open and challenging multiscale problem (see Section 6 for a discussion).

Within GQD, we introduce a simplified treatment of BH dynamics, encompassing light and heavy seeds and massive BHs, by attributing to triple interactions the role of taxing $\mathrm{BHs}$ down to coalescence. ${ }^{4}$ This is motivated by the high incidence of multiple mergers among DM haloes occurring at high redshift and traced by GQD. We adopt the model by Bonetti et al. (2016, 2018a, 2018b), who carried out a large suite of numerical simulations with a three-body postNewtonian code describing the mutual interaction among BHs over a wide range of masses, mass ratios, and orbit initial conditions, framed in spherical galactic potentials (Bonetti et al. 2016). Multiple $\mathrm{BH}$ encounters provide a viable solution to the so-called final parsec problem (i.e. the stalling of binaries at separations below $\sim \mathrm{pc}$ ) when all other shrinking mechanisms are not efficient (Bonetti et al. 2018a) and are expected to have an important role in SMBH evolution (as in our model) as well as on future GW detections in particular in the LISA band (Bonetti et al. 2019).

Hereon, we assume that a Keplerian BH binary forms promptly in a major halo merger, and that it is dragged in the nuclear region

\footnotetext{
${ }^{2}$ This is the volume of the $10^{13} \mathrm{M}_{\odot}$ DM halo computed at the turnaround radius.

${ }^{3}$ In this paper, major mergers refer to interacting DM haloes with mass ratios greater than $1: 4$.

${ }^{4}$ Actually, other physical mechanisms can influence the evolution of BHs, both before and after the pairing. See Section 6 for a discussion.
} 
Table 1. Properties of selected quasars and main free parameters of GQD models.

\begin{tabular}{lcccccc}
\hline Object & $z_{\mathrm{QSO}}$ & $\begin{array}{c}\mathrm{SFR} \\
\left(\mathrm{M}_{\odot} \mathrm{yr}^{-1}\right)\end{array}$ & $\log \left(M_{\mathrm{SMBH}} / \mathrm{M}_{\odot}\right)$ & $\epsilon_{\text {quiesc }}$ & $\alpha_{\mathrm{BH}}$ & $\epsilon_{\mathrm{AGN}, \mathrm{w}}$ \\
\hline $\mathrm{J} 1148$ & 6.4 & $100-1000$ & $9.5_{-0.2}^{+0.3}$ & 0.1 & 110 & $2 \times 10^{-3}$ \\
$\mathrm{~J} 2345$ & 2.0 & $50-330$ & $9.47 \pm 0.3$ & 0.5 & 50 & $2 \times 10^{-3}$ \\
PDS456 & 0.2 & $30-80$ & $9.4 \pm 0.17$ & 0.5 & 50 & $2 \times 10^{-3}$ \\
\hline
\end{tabular}

of the newly formed halo where it stalls until it interacts with a third incoming $\mathrm{BH}$, called an intruder. This implicitly assumes that, within at most a few Myr, the characteristic (redshift-dependent) time interval of our simulations, DM/stellar/gas dynamical friction is effective in forming a binary during the so-called pairing phase, when the BHs sink as individual masses inside the halo merger remnant (Begelman, Blandford \& Rees 1980). Indeed, a general expectation is that, at least when the two merging galaxies have mass ratios $>0.05-0.1$, dynamical friction efficiently drags the BHs from the outskirts towards the centre of the newly formed galaxy within about a few million (up to a billion) years (Mayer et al. 2007; Callegari et al. 2009; Van Wassenhove et al. 2014; Capelo et al. 2015; Khan et al. 2016; Biava et al. 2019).

Subsequently, the intruder dragged by a third incoming DM halo can then interact with the binary via chaotic strong triple encounters or by Kozai-Lidov evolution following the formation of a bound hierarchical triplet (Kozai 1962; Lidov 1962; Bonetti et al. 2016, 2018b).

In our model, the fate of a triplet is defined on the basis of the statistical study presented by Bonetti et al. (2018b). We use their results to distinguish triplets (potentially) leading to coalescence from systems that would never do (as their associated/computed merger time-scale is longer than the Hubble time at $z=0$ ). In this way, we are accounting for the (global) efficiency of triple interactions in driving BH mergers, limiting the fraction of events. Quadruple encounters are reduced to a three-body problem by means of the ejection of the lightest $\mathrm{BH}$ and iterated as triple systems (see Bonetti et al. 2018a, for details).

In a triple encounter, the pairs that eventually coalesce are selected on the basis of the merger fractions and relative occurrence probabilities computed by Bonetti et al. (2018b). We assign a probability to any pair of BHs in a triple merger and randomly extract the outcome of the interaction by interpolating through their model grid of primary $\mathrm{BH}$ masses $\left(m_{1}\right)$ and inner and outer mass ratios ( $q_{\text {in }}$ and $q_{\text {out }}$, respectively). ${ }^{5}$ These same properties also define the merger time-scale of each system. However, in our SAM we adopt a simplified assumption: In successful triplet-induced merger, the two BHs coalesce within the characteristic simulation time interval (up to few Myr). This assumption implies that BBH merger times are determined mainly by the sequence (rate) of $\mathrm{BH}$-seeded halo-halo encounters within a merger tree, rather than by dynamical processes. We will discuss this point in Section 6.

To summarize, in our model $\mathrm{BH}$, mergers are triggered only via triplet formation and triple interactions and have two possible outcomes: (i) the 'instantaneous' coalescence of any two BHs; and (ii) the formation of a so-called 'leftover' binary (no merger), with the ejection at larger scales of one of the involved BHs (usually the lighter).

\footnotetext{
${ }^{5}$ The probabilities of the closest grid point are assigned to triplets whose parameters are outside the range sampled by Bonetti et al. (2018b).
}

\section{THE EMERGENCE OF BINARY BLACK HOLES}

As mentioned in Section 2, the model reconstructs the formation histories of three luminous quasars at $z_{\mathrm{QSO}}=6.42 .0$ and 0.2 . We choose as proto-typical objects for the three redshifts the quasars J1148 at $z_{\text {QSO }}=6.4$ (Fan et al. 2001), SDSS J2345+1104 at $z=2$ (hereafter J2345; Shen et al. 2011; Schulze et al. 2019), and PDS 456 at $z=$ 0.2 (PDS456 hereafter; Nardini et al. 2015; Bischetti et al. 2019).

We model the evolution of J1148, J2345, and PDS456 performing, for each of them, 10 independent simulations adopting the set of model parameters described in Table 1 . These are tuned to reproduced the observed SMBH mass and host galaxy physical properties (see Valiante et al. 2011, 2014, 2016, for more details).

To investigate the emergence of $\mathrm{BBH}$ populations across the cosmic epochs (in our cosmological framework), we select one 'fiducial' simulation (out of the 10 performed) for each template quasar. In particular, in what follows we show the results of the simulation that provides a global SMBH evolution that best matches the corresponding simulation-averaged predictions.

\subsection{From seeds to binaries along a merger tree}

In Fig. 1, we show the distributions of light seeds (on the left-hand panel) and heavy seeds (on the right) as a function of their formation redshift. Grey, blue, and red histograms refer to quasars J1148, J2345, and PDS456, respectively.

The number of seeds and the shapes of the histograms are similar for the three quasars. The bi-modal distribution of light seeds reflects the properties of Pop III star-forming haloes. At early times, they are mainly mini-haloes where star formation is dramatically limited by radiative feedback $\left(\mathrm{H}_{2}\right.$ photodissociating radiation in particular). At later epochs, Pop III stars (and thus light seeds) instead mainly form in atomic cooling haloes that are less affected by the presence of external UV radiation (see Valiante et al. 2016, for a more detailed description).

On the other hand, heavy seeds form only in atomic cooling haloes and the environmental conditions required by the DCBH formation scenario (sub-critical metallicity and a supercritical illuminating LW radiation; see Section 2.3) are met only over a very limited period of time and by a limited, very low, number of haloes within our merger trees.

A total of 39 (31 and 40) heavy and 4228 (5327 and 5319) light seeds are formed along the assembly history of J1148 (J2345 and PDS456, respectively). In all cases, light seeds form in larger numbers at very high redshift $(12<z<30)$ and over a longer period of cosmic evolution than heavy seeds, which are rarer (with a relative fraction of $\sim 1$ per cent) and form for a shorter period of time at slightly lower redshift $(z \sim 12-17$, depending on the considered system).

In-situ and/or external pollution determines the end of the seed (and Pop III stars) formation era: As soon as all the galaxies have been enriched above the critical metallicity threshold $\left(Z_{\mathrm{cr}}=10^{-3.8} \mathrm{Z}_{\odot}\right)$, 

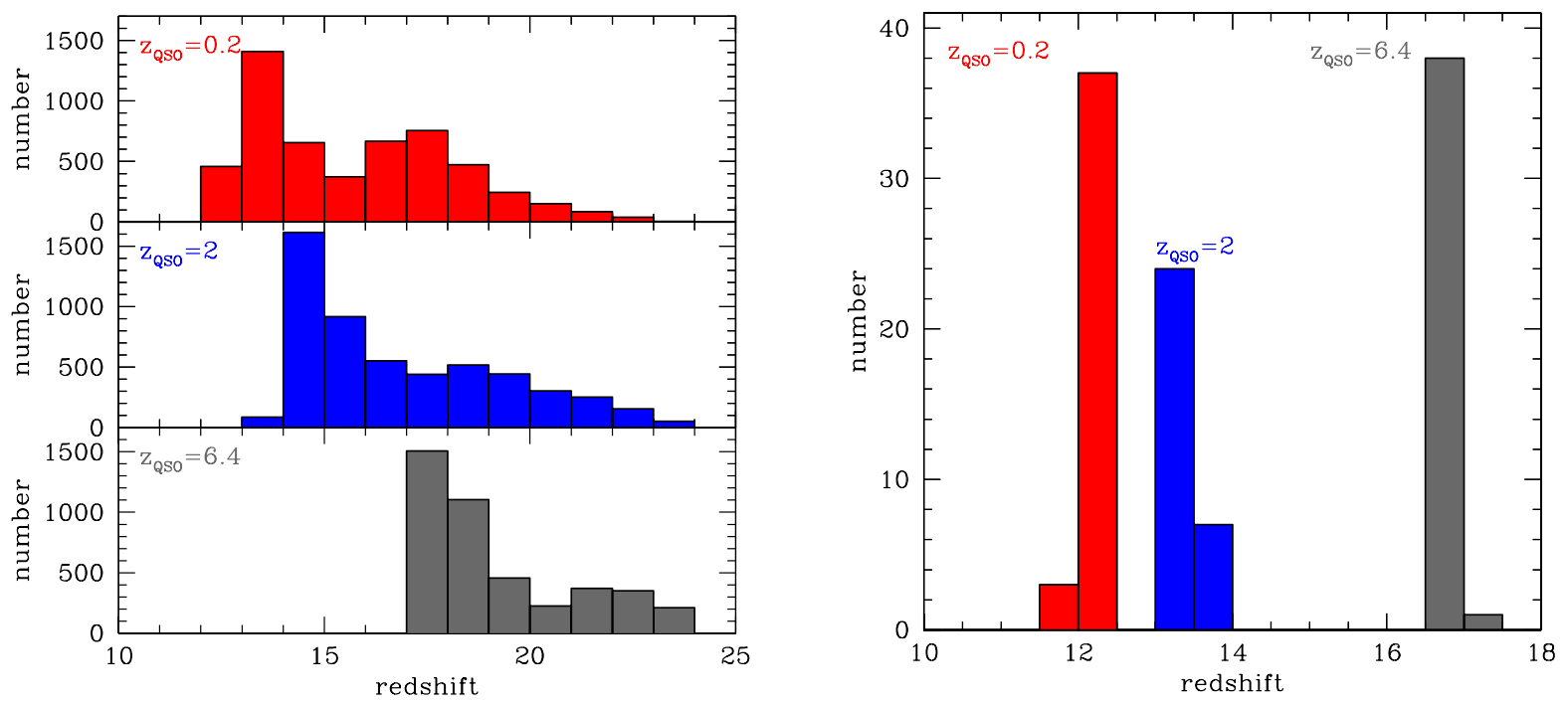

Figure 1. Redshift distribution of the number of light (left-hand panel) and heavy (right-hand panel) seed BHs forming along the 'fiducial' merger history of quasar J1148 outshining at $z_{\mathrm{QSO}}=6.4$ (grey histograms), $\mathrm{J} 2345$ at $z_{\mathrm{QSO}}=2$ (blue histograms), and PDS456 at $z_{\mathrm{QSO}}=0.2$ (red histograms).
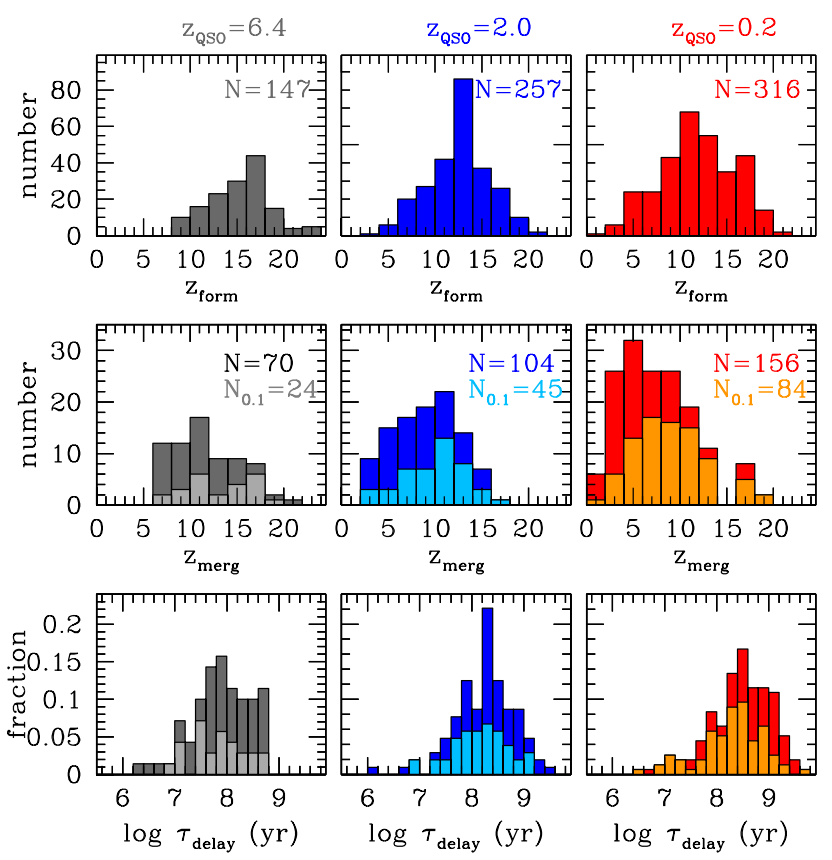

Figure 2. Upper panels: the number of BBHs as a function of their formation redshift, $z_{\text {form }}$, in quasar models for J1148 (grey histogram on the left), J2345 (blue histogram, in the middle), and PDS456 (red histogram, on the right). Labels in each panel indicate the total number of binaries that form, summed over all redshifts. Central panels: distribution of the number of triplet-driven merging $\mathrm{BHs}$ at their merger redshift, $z_{\text {merg }}$, for the same three quasars. The total numbers of $\mathrm{BH}$ coalescences are labelled in dark in each panel. Bottom panels: distribution of BBH merger time delays, $\tau_{\text {delay }}$ (or lifetimes; see the text for details). In central and bottom panels, lighter colours show distributions for merging binaries with a mass ratio $q \geq 0.1$.

the transition to the Pop II star formation regime is completed. This critical level is reached, on average, at $z \sim 16,13$, and 12 for quasars J1148, J2345, and PDS456, respectively. Below this redshift, light and heavy seeds no longer form.
For each of the three simulations, Fig. 2 shows the number of BBHs at their formation redshift, $z_{\text {form }}$ (upper panels), and the number of merging $\mathrm{BBHs}$ at their coalescence redshift $z_{\text {merg }}$ (central panels). The latter is the redshift at which a triplet $\mathrm{BH}$ system forms, leading to the prompt coalescence of a $\mathrm{BBH}$, according to the physical prescriptions described in Section 3.

In the upper panel of Fig. 2, we show that $147(257,316)$ binaries form over the simulated cosmic time, $2900 \mathrm{Myr}$ (3 and $11 \mathrm{Gyr}$ ) for quasar J1148 (J2345 and PDS456, respectively). ${ }^{6}$

The histograms in the bottom panels of Fig. 2 show the distribution of the delay times to coalescence, $\tau_{\text {delay }}$. In our model, the merger time-scale of two BHs, following halo assembly, corresponds to the time elapsed from the formation of the $i$-th binary down to coalescence, driven by a successful multiple $\mathrm{BH}$ interaction, involving that binary, i.e. $\tau_{\text {delay, } \mathrm{i}}=t\left(z_{\text {merg, }, \mathrm{i}}\right)-t\left(z_{\text {form, } \mathrm{i}}\right)$. The mean values of the delay time distributions are of the order of $\sim 150,360$, and $590 \mathrm{Myr}$, respectively, in the simulations of quasars like J1148 $\left(z_{\mathrm{QSO}}=6.4\right)$, $\mathrm{J} 2345\left(z_{\mathrm{QSO}}=2\right)$, and PDS456 $\left(z_{\mathrm{QSO}}=0.2\right)$.

These delays correspond to the typical time-scales of triple halo interactions, each hosting a nuclear BH. Two additional delay times should be considered: the formation time-scale of the binary system (i.e. the time required for the nuclear $\mathrm{BHs}$ of the merging haloes to reach the centre of the newly formed system and to dynamically pair) and the time required for the system to coalesce. These timescales are not considered in this study. A detailed discussion will be presented in Section 6.

Finally, histograms drawn in lighter colours in the central and bottom panels of Fig. 2 represent the distributions of BBHs with a mass ratio $q \geq 0.1$, whose $\mathrm{GW}$ emission will be analysed in the next section.

\footnotetext{
${ }^{6}$ Each simulation of a quasar is characterized by its peculiar number of seeds and halo major mergers. For example, the number of heavy seeds that form across cosmic times, along a merger tree, can vary from a few up to few tens, depending on the specific simulation, mirroring the relative efficiency of chemical and radiative feedbacks in each history (see Valiante et al. 2016, for a discussion). However, we find that the redshift intervals over which the seeds and BBHs form and merge, as well as the merger time-scale distribution, are very similar, i.e. do not vary much, among the different simulations of a given quasar
} 


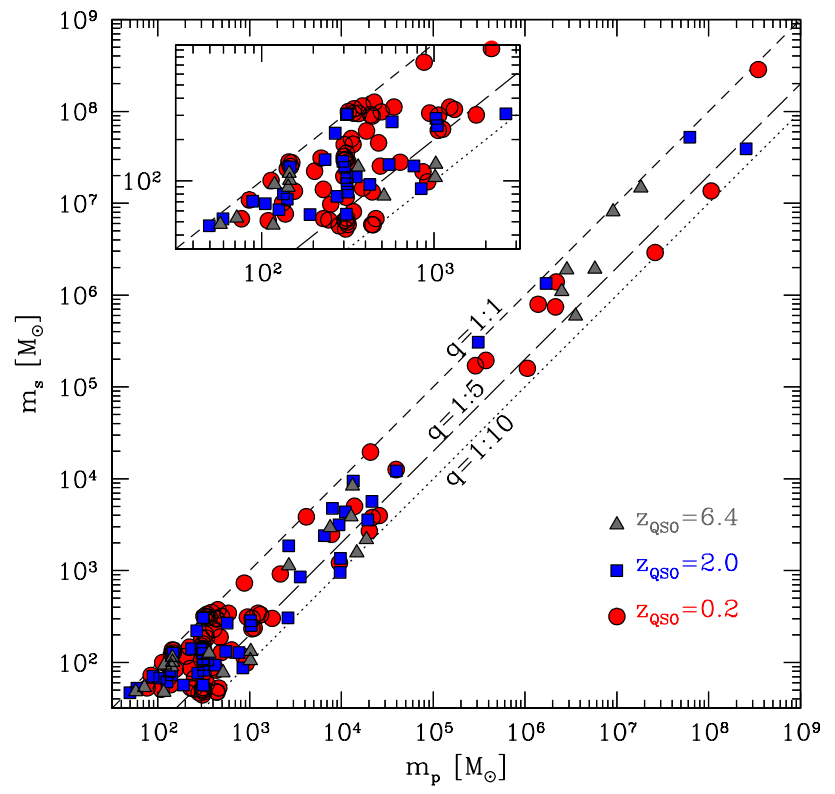

Figure 3. Mass of the primary $\left(m_{\mathrm{p}}\right)$ and secondary $\left(m_{\mathrm{s}}<m_{\mathrm{p}}\right)$ components of merging BBHs with a mass ratio of $q=m_{\mathrm{s}} / m_{\mathrm{p}} \geq 0.1$, formed along the evolutionary history of the three quasars: $\mathrm{J} 1148$ at $z_{\mathrm{QSO}}=6.4$ (grey triangles), $\mathrm{J} 2345$ at $z_{\mathrm{QSO}}=2$ (blue squares), and PDS456 at $z_{\mathrm{QSO}}=0.2$ (red circles). Dashed, log-dashed, and dotted lines mark secondary over primary mass ratios equal to $1: 1,1: 5$, and $1: 10$, respectively. The inserted box on the top left zooms on $<10^{3} \mathrm{M}_{\odot}$ binaries.

\subsection{Merging BHs in the ET and LISA frequency domains}

In this section, we describe the properties of coalescing BBHs extracted from GQD for each of the three quasars. Then, we discuss their detectability in the ET high-frequency and LISA low-frequency domains.

The primary (most massive) and secondary $\mathrm{BH}$ masses in merging $\mathrm{BBH}$ systems are shown in Fig. 3. Given the wide mass interval probed by GQD, haloes are found to host dual/multiple BHs with mass ratios as small $10^{-2}-10^{-4}$ for which we could not follow their as yet unknown (likely erratic) dynamics. Binaries with such small mass ratios might never form as a consequence of the long dynamical friction time-scale (e.g. Dosopoulou \& Antonini 2017). For this reason, we do not include these systems in our analysis and the figure reports BBHs with a mass ratio of $q \equiv m_{\mathrm{s}} / m_{\mathrm{p}}>0.1$, which cover almost uniformly the $0.1 \leq q \leq 1$ interval.

In Fig. 4, we show the distribution of $\mathrm{BH}$ mergers in the $z-m_{\mathrm{BH}, \mathrm{T}}$ plane, where $m_{\mathrm{BH}, \mathrm{T}}$ is the total mass of the binary in the source rest frame. Different symbols/colours pinpoint cosmologically driven $\mathrm{BBH}$ coalescences triggered by triple interactions that occur during the assembly of the three simulated quasars: J1148 (grey triangle, $z_{\mathrm{QSO}}=6.4$ ), $\mathrm{J} 2345$ (blue square, $z_{\mathrm{QSO}}=2$ ), and PDS456 (red circle, $z_{\mathrm{QSO}}=0.2$ ). Data points with white edges indicate mergers involving at least one heavy seed.

Overlaid in Fig. 4 are contour lines of constant signal-to-noise $(\mathrm{S} / \mathrm{N})$ ratio computed using the ET-D sensitivity curve by Hild et al. (2011) for ET, and that of Robson, Cornish \& Liu (2019) for LISA. The IMRPhenomC (Santamaría et al. 2010) gravitational waveform family is used to compute the strength of the signal assuming nonspinning BHs, which includes only the 22 quadrupolar mode. The ensemble of colour-coded areas for a given detector is often referred to as 'waterfall' plot that provides values of the $\mathrm{S} / \mathrm{N}$ ratio at which a GW source would be detected, averaged over the source's sky position, and the binary-inclination and GW-polarization angles. ${ }^{7,8}$

The figure shows that both observatories shall have the capability of detecting GWs from coalescences occurring at redshifts as large as $z \sim 15$ (and even beyond for a narrower interval of masses), letting us explore the epochs of seed formation and growth. But not only that, coalescence events are found to spread over a much wider range in redshift and mass: down to $z \simeq 2$ and up to a few $10^{7} \mathrm{M}_{\odot}$. The lack of mergers at very low redshifts is a consequence of our model assumptions.

In more detail, Fig. 4 shows how densely populated are the two GW windows during the cosmic assembly of our simulated quasars. The fastest evolution is associated with the $z_{\mathrm{QSO}}=6.4$ quasar. Here, the galaxy haloes and $\mathrm{BHs}$ evolve at a rapid pace and the associated GW events drift away from the ET bandwidth swiftly, most of them transiting across the deci-Hz window (Sato et al. 2017; Arca Sedda et al. 2019a) already at $z>12$. For this quasar model (J1148), a few coalescences of BBHs involving pairs of light seeds would be visible in the ET band at $z=14-16$. However, most of the events involving BHs grown from light seeds occurring mainly at $z \geq 12$ would be visible in the LISA band when the BHs have achieved masses of $\sim 10^{4} \mathrm{M}_{\odot}$, due to efficient gas accretion in the environments. We have to wait until redshift $z \sim 10-11$ to see a $q>0.1$ merger involving at least one BH grown from a heavy seed. The bulk of these heavyseed mergers (three involving two BHs grown from heavy seeds and four with $\mathrm{BH}$ pairs grown from a light seed and a heavy seed) occur between $8<z<11$, when the Universe is only $600 \mathrm{Myr}$ old.

Despite our results being based on a limited number of trials, we do find that, generically, mergers (with $q>0.1$ ) involving BHs that originate from heavy seeds appear in the LISA band when the original seeds have already increased their mass by gas accretion up to $M_{\mathrm{BH}} \geq 10^{6} \mathrm{M}_{\odot}$. A similar trend is also observed in the BBHs formed along the evolution of the $z_{\mathrm{QSO}}=2$ and 0.2 quasars. In the latter case, $\sim 20$ per cent of all detectable mergers involving heavy seeds are found in the mass range $10^{5}<m_{\mathrm{BH}, \mathrm{T}} / \mathrm{M}_{\odot}<10^{6}$. We warn, however, that if heavy seeds were to form with a wider mass spectrum than that considered in our model, extending from less than $10^{4} \mathrm{M}_{\odot}$ up to a few $10^{5} \mathrm{M}_{\odot}$, the mid region of the LISA band would also be populated of events.

In our model, the assembly of a quasar at redshift $z_{\mathrm{QSO}}=6.4$ constrains the flow of data points across the $z-m_{\mathrm{BH}}$, $\mathrm{T}$ plane. It acts as a terminal point of the cosmological evolution of $\mathrm{BH}$ seeds. Detecting a coalescence at redshift as large as $z \sim 10-14$ with ET and a coalescence just on the edge of the left side of the LISA waterfall plot at adjacent redshifts would provide the first evidence that light seeds form and grow via accretion in high- $z$ gas-rich environments, and are dynamically paired in coalescing binaries during galaxy mergers.

Yet, the detection of these events is challenging. In ET, they mainly lie in the declining (right) side of the waterfall envelope and are characterized by low S/N ratios (Kalogera et al. 2019). Here, the portion of the detected GW signals traces only at most 1-2 cycles of the inspiral, and the merger and ringdown. On the other hand,

${ }^{7} \mathrm{~A} \mathrm{~S} / \mathrm{N}$ threshold between 5 and 10 is customarily taken as detection threshold for any $\mathrm{GW}$ event. Here, we consider $\mathrm{S} / \mathrm{N}=10$ as the detection threshold. ${ }^{8} \mathrm{CE}$ and ET will be part of a network of detectors that will enlarge the GW cosmic horizon. Although they will have comparable sensitivities, ET will be more sensitive below $10 \mathrm{~Hz}$, with $\mathrm{CE}$ more sensitive at higher frequencies. Consequently, ET will have better sensitivity to higher mass mergers $\left(\gtrsim 100 \mathrm{M}_{\odot}\right)$, with CE being more sensitive at lower masses $\left(\lesssim 20 \mathrm{M}_{\odot}\right.$; Hall \& Evans 2019). As the focus of this study is on binaries above $100 \mathrm{M}_{\odot}$, we only show the sensitivity of ET in Figs 4, 5, and 8. 


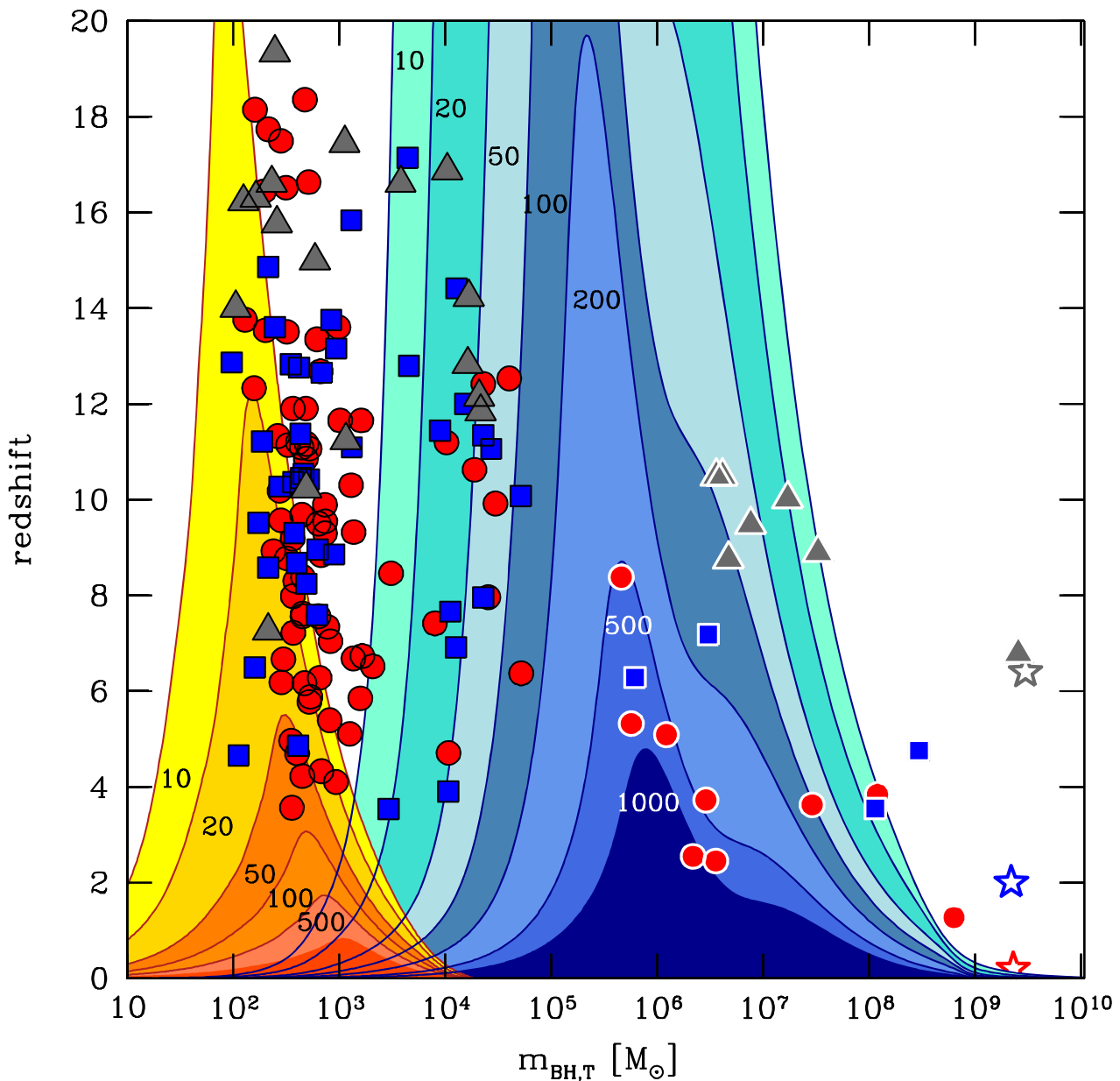

Figure 4. Distribution of $\mathrm{BBH}$ coalescence events in the redshift $z-m_{\mathrm{BH}} \mathrm{T}$ diagram. Data points describe cosmologically driven BH mergers with a mass ratio $q \geq 0.1$, triggered only by triple interactions among galaxy haloes. Grey triangles, blue squares, and red circles denotes the total mass and redshift of the coalescences extracted from the simulation of a $10^{13} \mathrm{M}_{\odot}$ overdensity, forming an $\sim 10^{9} \mathrm{M}_{\odot} \mathrm{SMBH}$ at $z_{\mathrm{QSO}}=6.4,2$, and 0.2 (represented with stars in the plot). Symbols with white edges indicate mergers involving at least one heavy seed. Colour-coded areas represent lines of constant S/N ratios for ET (yellow/red) and LISA (azure/blue) computed for non-spinning binaries assuming a mass ratio of $q=0.5$, which corresponds to the mean value of the merging binaries extracted from our samples. The ensemble of the colour-coded areas for a given detector is often referred to 'waterfall' plot and provides averaged values of the $\mathrm{S} / \mathrm{N}$ ratio at which a GW source is detected.

the coalescences of light seeds grown up to masses of $10^{3}-10^{4} \mathrm{M}_{\odot}$ at $z$ larger than 10 lie in the rising side of the waterfall envelope in the LISA band, and are far louder GW sources, with $\mathrm{S} / \mathrm{N}$ ratios in the range 10-50. The GW signal takes now the shape of a nearly adiabatic inspiral, as their merger falls in the deci-Hz window.

Coalescences that involve heavy seeds in the LISA band at $z \sim 10$ are at the edge of the declining side of the waterfall plot, and their GW signal (with $\mathrm{S} / \mathrm{N}$ ratios in the [10-100] interval) is dominated again by few cycles in the inspiral, and by the merger and ringdown phases.

Due to the incompleteness of our modelling, we cannot exclude the presence of evolved seeds of $10^{5} \mathrm{M}_{\odot}$ up to a few $10^{6} \mathrm{M}_{\odot}$ that will be observable as high-S/N GW sources in LISA.

The assembly histories of the two remaining quasars have as anchor points of the simulations two lower redshift systems, and as a consequence coalescences are distributed over a wider redshift interval, implying the appearance of lower $z$, louder GW sources, both in the ET and LISA frequency domains.

Considerations similar to those discussed for the highest redshift quasar simulation apply here. However, here we clearly see that, besides the population of BBHs swiftly transiting to higher masses (to enable the formation of an SMBH of $\sim 10^{9} \mathrm{M}_{\odot}$ ), there exists a lower redshift population, which we call 'starved' binary seeds, with masses in the range between $100 \mathrm{M}_{\odot}$ and a few $10^{3} \mathrm{M}_{\odot}$. These systems are hosted by haloes where seeds were unable to grow or that grew only marginally, filling the middle weight mass range. Also, the number of $\mathrm{BH}$ mergers increases, reflecting the larger number of progenitor haloes (and thus halo-halo coalescences) in the merger trees of the lower $z$ simulated quasars (see Section 2.1): While we witnessed 24 mergers in the $z_{\mathrm{QSO}}=6.4$ halo, in the $z_{\mathrm{QSO}}=2$ and 0.2 haloes, we have 45 and 84 mergers, respectively, with only a handful (less than 10 per cent, on average) comprising heavy seeds.

\subsubsection{The impact of cosmic variance}

Although we analyse here a single simulation of each quasar, the findings discussed above do not dramatically depend on the selected simulation. The dispersion in the $z-m_{\mathrm{BH}, \mathrm{T}}$ plane due to the choice of a specific merger tree simulation can be appreciated in Fig. 5 where we collect BH mergers, with $q>0.1$, extracted from five realizations of each quasar. 


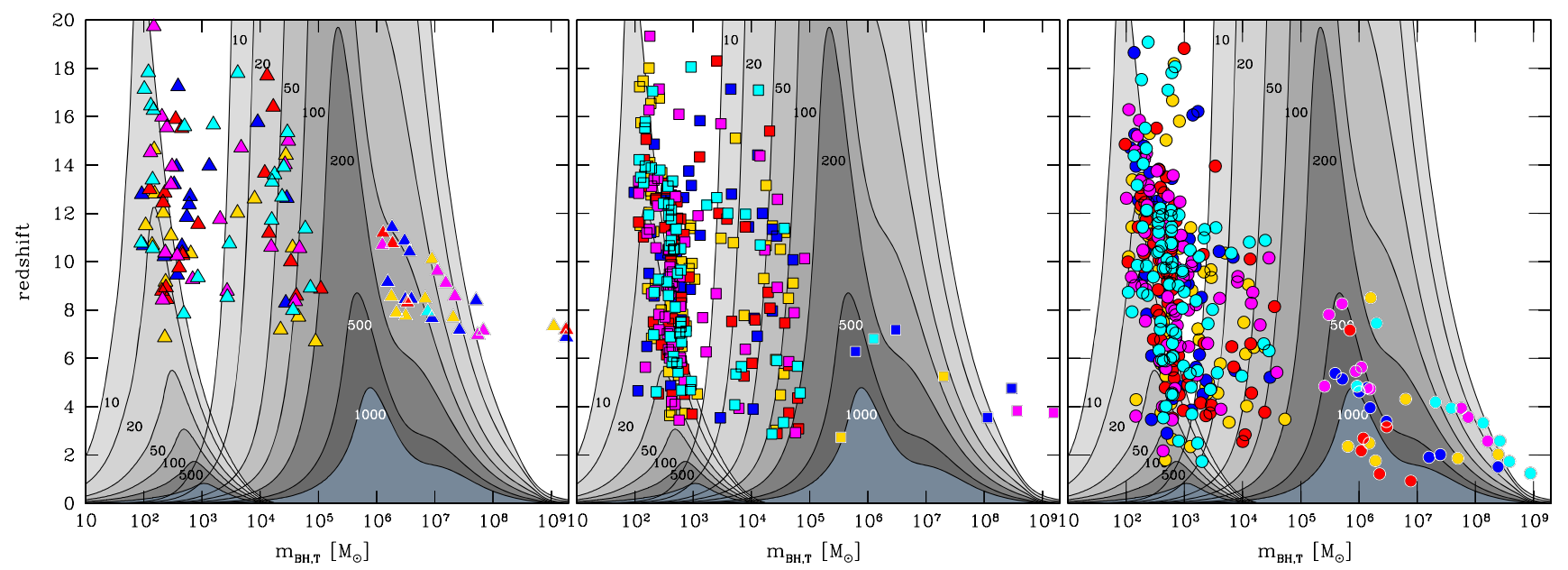

Figure 5. Same as in Fig. 4 but comparing five simulations of each quasar at $z_{\mathrm{QSO}}=6.4$ (left-hand panel), 2 (central panel), and 0.2 (right-hand panel). In all the panels, different colours indicate BH coalescences extracted from a given merger tree simulation of the same considered quasar. The ET and LISA Waterfall plots are shown in different shades of grey, for simplicity.

Table 2. Statistical analysis of $\mathrm{BH}$ mergers: the number of $\mathrm{BH}$ mergers with a mass ratio $q \geq 0.1\left(n_{0.1}\right)$ and the fraction of these coalescences involving pairs of light $\left(f_{\mathrm{L}-\mathrm{L}}\right)$, light + heavy $\left(f_{\mathrm{L}-\mathrm{H}}\right)$, and heavy $\left(f_{\mathrm{H}-\mathrm{H}}\right)$ seeds. The upper table refers to the single 'fiducial' realizations of each simulated quasar while mean values, averaged over 10 merger histories for each system, are reported in the bottom table.

\begin{tabular}{|c|c|c|c|c|}
\hline$z_{\mathrm{QSO}}$ & $n_{0.1}$ & $f_{\mathrm{L}-\mathrm{L}}$ & $f_{\mathrm{L}-\mathrm{H}}$ & $f_{\mathrm{H}-\mathrm{H}}$ \\
\hline \multicolumn{5}{|c|}{ Single simulation } \\
\hline 6.4 & 24 & 71 per cent & 17 per cent & 13 per cent \\
\hline 2.0 & 45 & 91 per cent & 7 per cent & 2 per cent \\
\hline 0.2 & 84 & 89 per cent & 11 per cent & 0 \\
\hline \multicolumn{5}{|c|}{ Simulations averaged } \\
\hline 6.4 & 27 & 86 per cent & 12 per cent & 2 per cent \\
\hline 2.0 & 62 & 97 per cent & 2.4 per cent & 0.6 per cent \\
\hline 0.2 & 78 & 89 per cent & 8.3 per cent & 2.5 per cent \\
\hline
\end{tabular}

For the $z_{\mathrm{QSO}}=6.4$ quasar, the choice of the merger history mainly affects the fraction of mergers (involving pairs of light seeds) that could be detected in the ET band at $z<12$. This varies from 18 per cent (magenta triangles) up to $\sim 35$ per cent (cyan triangles); 24 per cent is found for the realization shown in Fig. 4. The redshift distribution of starved binaries extends towards lower redshifts (down to $z \sim 2$ ) when different formation histories are considered for the $z_{\mathrm{QSO}}=0.2$ quasar. Within our model, we can investigate the relative occurrence of binary coalescences involving BHs of different origins, along the quasar evolution history. The vast majority of BH mergers ( $\sim 90$ per cent) involve pairs of light seeds, as they are more common than heavy seeds as shown in Fig. 1. The fraction of mergers involving the two seed flavours is reported in Table 2.

The merging binaries in our models could be 'multiband sources', i.e. sources that transit from the LISA low-frequency domain (during their long-lived inspiral phase) to the ET/CE high-frequency domain (merger and ringdown) if their lifetime in the LISA band is shorter than the nominal lifetime of the mission (4-10 yr). Joint multiband observations of the same event will be possible for $\left(10^{2}-10^{4}\right) \mathrm{M}_{\odot}$ BBHs out to redshifts of 4-5 (e.g. Jani, Shoemaker \& Cutler 2019). Multiband detections of distant lower (higher) mass binaries would be instead limited by the sensitivity for LISA at frequencies around and above $0.1 \mathrm{~Hz}$ (for ET/CE at frequencies around and below $3 \mathrm{~Hz}$ ).
We note here further that very few mergers in the $z_{\mathrm{QSO}}=0.2$ quasar model (right-hand panel of Fig. 5) are predicted to be observable both in ET and LISA (none in the 'fiducial' model shown in Fig. 4).

We further remark that coalescing stellar BBHs, relic of massive population III stars, could also form in situ (Hirano et al. 2018; Sugimura et al. 2020). These non-cosmologically driven mergers are not included in the figure, nor the population of binaries forming via dynamical captures in dense environment such as young star clusters (e.g. Di Carlo et al. 2020) and globular clusters (e.g. Rodriguez, Chatterjee \& Rasio 2016; Askar et al. 2017) or in galactic fields via ordinary channels (e.g. Dominik et al. 2012, 2013, 2015; Mapelli et al. 2017; Mapelli \& Giacobbo 2018; Mapelli et al. 2019), particularly the most massive ones (Schneider et al. 2017; Marassi et al. 2019; Graziani et al. 2020). We expect that these stellar BHs will preferentially fill the left corner of the ET waterfall plot, as shown later in Fig. 8, extending out to the redshifts at which star formation started (Santoliquido et al. 2020).

\subsubsection{Event rates in the LISA and ET sky}

In principle, to compute the total number of detectable sources per year, namely the event rates in the LISA and ET band, we would need to simulate a large number of merger trees, spanning a wide range of parent halo masses and formation redshifts (weighting each mass according to the expected halo mass function). Nevertheless, at $z \sim$ 0 DM haloes of $10^{13} \mathrm{M}_{\odot}$ are expected to be common; thus, using our $z_{\mathrm{QSO}}=0.2$ model as representative of an 'average Universe' would provide a reasonable estimate of the merger rates. ${ }^{9}$

To this aim, we first compute the intrinsic rates and chirp masses of the $\mathrm{BH}-\mathrm{BH}$ mergers extracted from our 10 realizations of the $z_{\mathrm{QSO}}$ $=0.2$ quasar model. ${ }^{10}$

\footnotetext{
${ }^{9}$ The $z_{\text {QSO }}=6.4$ and 2 predictions would provide extremely incomplete estimates of the detectable event rates as, at those redshifts, DM haloes of $10^{13} \mathrm{M}_{\odot}$ are instead the highest $\sigma$ mass density fluctuations, representative of highly biased regions of the Universe.

${ }^{10}$ Using the average comoving volume occupied by a typical $10^{13} \mathrm{M}_{\odot}$ halo $\left(\sim 300 \mathrm{Mpc}^{3}\right)$ to weight our intrinsic merger rates, we find a simulationsaveraged total value of $\sim 83$ mergers per year $(\sim 93$ per year using the 'fiducial' simulation presented in Fig. 4).
} 

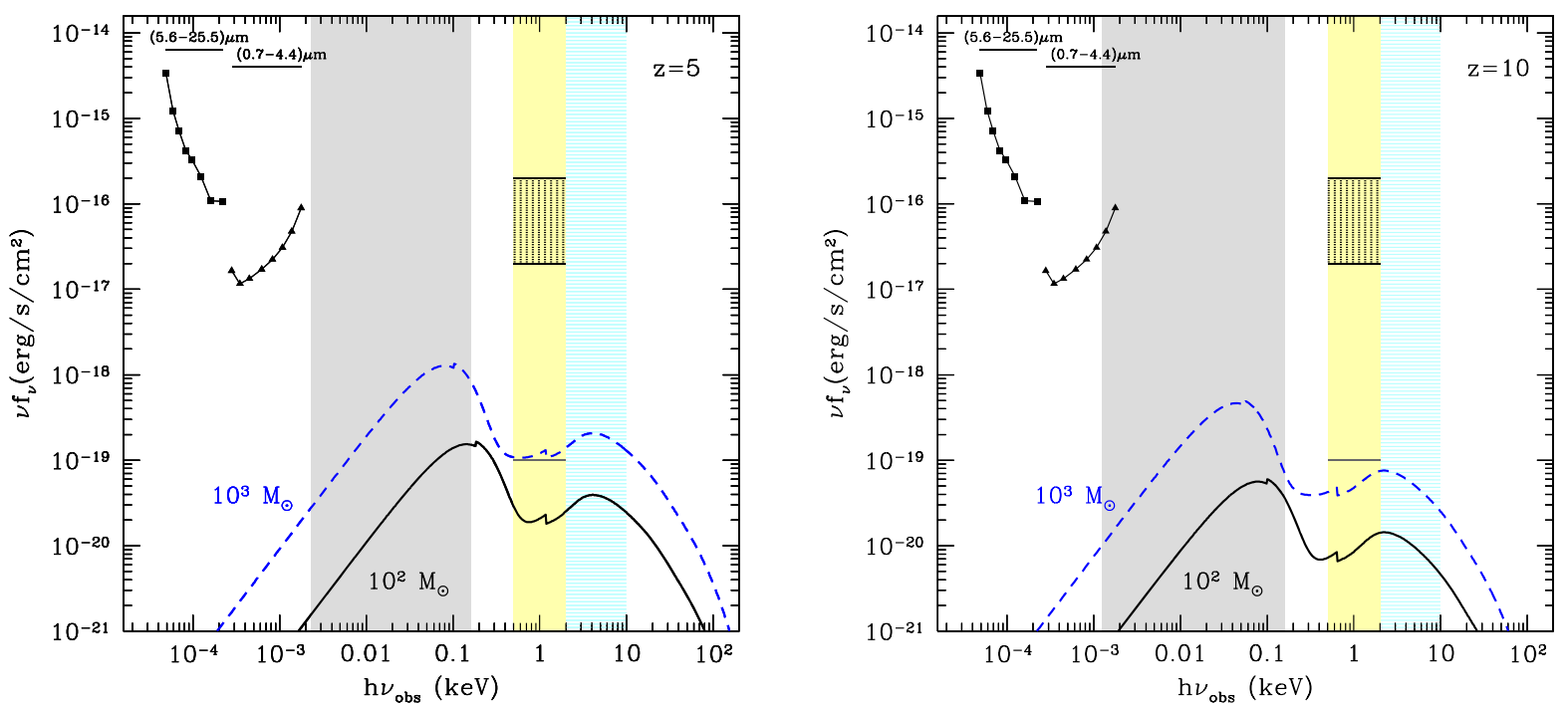

Figure 6. SED in the observer frame of two light seeds with masses of 100 and $1000 \mathrm{M}_{\odot}$ accreting at the Eddington limit under the most optimistic assumption for detectability, i.e. negligible obscuration and lack of stellar optical/UV emission. We show the SEDs at two different redshifts: $z=10$ (left-hand panel) and 5 (right-hand panel). Black lines with points show the sensitivity limits of NIRcam (triangles) and MIRI (squares), onboard of JWST, for a $10 \mathrm{ks}$ exposure. Hard and soft X-ray bands are marked by the cyan and yellow regions, respectively. The rectangle in the soft band shows the Athena area-dependent sensitivity range for the survey designed by Aird et al. (2013). The horizontal line marks the limiting sensitivity of Lynx in the soft X-ray for a point source of known position. Finally, the grey shaded area in both the panels indicates those wavelengths where emission is expected to be (almost completely) absorbed by the intervening neutral hydrogen along the line of sight.

We use these pieces of information to generate a Monte Carlo sample of all mergers occurring in $100 \mathrm{yr}$. Then the $\mathrm{S} / \mathrm{N}$ of simulated binaries is determined using the IMRPhenomC (Santamaría et al. 2010) waveforms with the corresponding sensitivity curves adopted in Fig. 4. For each binary, we randomized over sky position, inclination, and polarization in order to compute the fraction of detected sources. Assuming that only sources with $\mathrm{S} / \mathrm{N}>12$ (8) can actually be detected by ET (LISA), we obtain a total of 11.25 (18.7) events per year. The event rates in the LISA band are comparable to those obtained in other studies (e.g. Ricarte \& Natarajan 2018; Bonetti et al. 2019; Dayal et al. 2019).

Note, however, that populating the Universe only with $10^{13} \mathrm{M}_{\odot}$ haloes may lead to an overestimation of the number of events per year, when compared with merger rates weighted appropriately on the PS halo mass function. By extracting the merger rates for $10^{13} \mathrm{M}_{\odot}$ haloes and for the PS-weighted halo population from the model of Barausse (2012), we find the results to differ by a factor of $\lesssim 2.5$. Therefore, although crude, our estimate should be reliable within a factor of $\approx 2-3$.

Merger rates $\lesssim 1$ per year are instead obtained from the $z_{\mathrm{QSO}}=$ 2 and 6.4 models, normalizing the intrinsic rates to the observed number density of bright quasars at those redshifts $\left(\sim 10^{-7}\right.$ and $\sim 10^{-9} \mathrm{Mpc}^{-3}$, respectively). This suggests that $z=2$ and 6.4 quasars would contribute only a small fraction to the overall observed rate.

We stress here that computing actual/realistic merger rates is not one of the goals of this work, but will be the focus of future, improved, studies.

\section{OBSERVING THE EARLIEST ACCRETING BLACK HOLES WITH ELECTROMAGNETIC WAVES}

To date, EM signals from the earliest accreting BHs (seeds) at redshift $z>7.5$ are still missing. Although the Subaru High- $z$ Exploration of Low-Luminosity Quasars project enabled us to sample the faint-end tail of the $z \sim 6$ AGN luminosity function, down to a rest-frame ultraviolet absolute magnitude of $M_{1450}=-22 \mathrm{mag}\left(L_{\mathrm{bol}} \sim 10^{42}\right.$ $\mathrm{erg} \mathrm{s}^{-1}$; Matsuoka et al. 2018), no observational signatures of fainter AGNs, possibly powered by BHs of $\lesssim 10^{7} \mathrm{M}_{\odot}$, have been found at higher redshift.

If the high- $z$ population of fainter AGN is powered by heavy, growing seeds, current failed detections might be attributed to the low occupation fraction of this class of $\mathrm{BHs}$, mirroring the rare environmental conditions required for the $\mathrm{DCBH}$ formation. On the other hand, if the growing seed population is dominated by super-Eddington accreting light seeds (Inayoshi et al. 2017; Pezzulli et al. 2017b), the lack of detection could be due to their short and intermittent activity that is hard to capture within the limited sky coverage of current surveys (Pezzulli et al. 2017a).

In addition, X-ray observations of distant, lower mass (< $10^{5-6} \mathrm{M}_{\odot}$ ) faint AGNs are challenging as they may be hidden behind the radiation emitted by stellar X-ray binaries forming in the host galaxy, and may suffer from intrinsic obscuration (e.g. Volonteri et al. 2017).

With the next generation of facilities, such as Athena, early accreting BHs will be within reach, when searched in multitiered survey for an observing time of $25 \mathrm{Ms}$. The maximum redshift, compatible with the limiting sensitivity of the Wide Field Imager (WFI), is $z \leq 8$. Observations will provide lower limits on the $\mathrm{BH}$ masses, estimated to lie above $10^{6} \mathrm{M}_{\odot}$ (Aird et al. 2013). Lynx $x^{11}$ is a mission concept to explore the deep X-ray Universe, and in longexposure, multitiered surveys it is expected to discover the earliest BHs of $\sim 10^{4} \mathrm{M}_{\odot}$ out to $z \sim 10$.

In Fig. 6, we show the spectral energy distribution (SED) of light seeds of $10^{2}$ (solid lines) and $10^{3} \mathrm{M}_{\odot}$ (dashed lines) at redshift $z$ $=5$ (left-hand panel) and 10 (right-hand panel). In order to set the most favourable conditions for the detectability of unobscured,

\footnotetext{
${ }^{11} \mathrm{https} / / /$ wwwastro.msfc.nasa.gov/lynx/docs/LynxInterimReport.pdf
} 


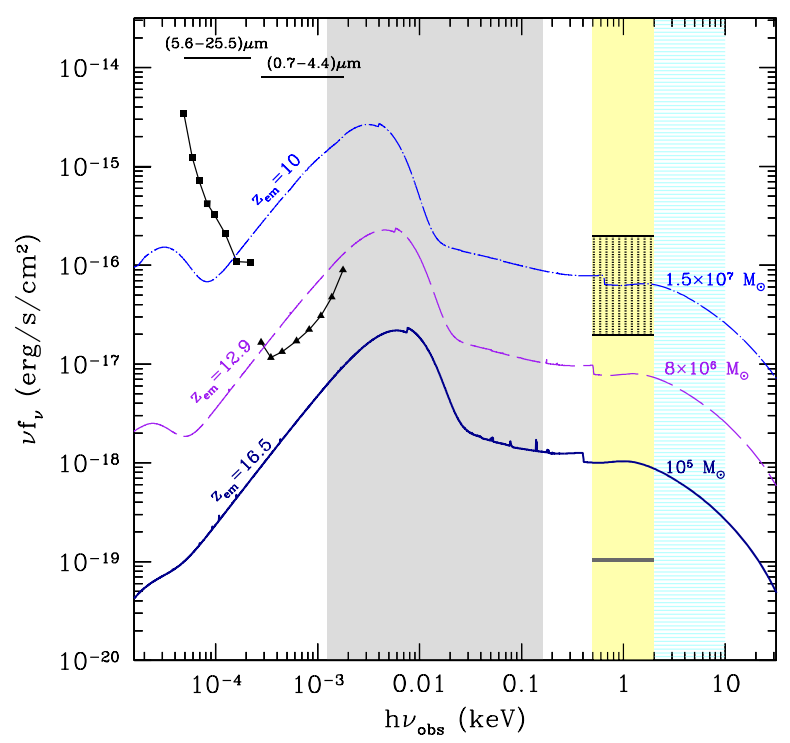

Figure 7. Time-dependent SEDs in the observe frame of a growing heavy seed forming at $z=16.5$ during the assembly of the $z_{\text {QSO }}=6.4$ quasar. The SED of the system is dominated by the emission of the accreting BH. Starting from an initial mass of $10^{5} \mathrm{M}_{\odot}$, the emission of a heavy seed is shown at three different ages, labelled with their emission redshift. At $z=10$, the $\mathrm{BH}$ mass is $1.5 \times 10^{7} \mathrm{M}_{\odot}$. The shaded areas, and the JWST, Athena, and Lynx limiting sensitivities are indicated as in Fig. 6 . The grey shaded region here shows the wavelength range affected by absorption along the light of sight for the source at $z=10$.

luminous light seeds, BHs are assumed to grow via gas accretion at the Eddington rate and the emission from the host galaxy (stellar component) as well as the photoelectric absorption from intervening neutral hydrogen have been neglected.

The SED comprises the optical/UV emission from a (standard) disc multicolour blackbody spectrum, and the X-ray emission from the hot corona, modelled as a power law with an exponential cut-off at a rest-frame photon energy of $300 \mathrm{keV}$ (see Pezzulli et al. 2017a; Valiante et al. 2018b, for details). The energy index of the power law in the $2-10 \mathrm{keV}$ interval is correlated with the Eddington ratio $\left(\lambda_{\mathrm{Ed}}\right)$ as $\Gamma=0.23 \log \lambda_{\mathrm{Ed}}+2.27$ (Brightman et al. 2013).

Modelled fluxes are compared with flux limits of different observatories/missions. Black lines with points show the sensitivity of the JWST (photometric) instruments NIRcam (triangles, 0.7-4.4 $\mu \mathrm{m}$ ) and MIRI (squares, 5.6-25.5 $\mu \mathrm{m}$ ) for a $10 \mathrm{ks}$ exposure. The limiting sensitivity of the concept Lynx, for a point source of known position, ${ }^{12}$ and the Athena area-dependent flux limit range for the survey designed by Aird et al. (2013) ${ }^{13}$ are shown as grey horizontal line and rectangle, respectively.

Fig. 6 shows that light seeds (in this vanilla model) would be too faint to be detectable at $z>5$ with next-generation EM facilities. Both the UV flux and the emission from the hot corona are below detectability, even considering the extreme capabilities of Lynx.

Following Valiante et al. (2018b), we also show in Fig. 7 the evolving SED of a heavy seed that forms at $z=16.5$ among the progenitors of the $z_{\mathrm{QSO}}=6.4$ quasar, and that grows via

\footnotetext{
${ }^{12}$ https://wwwastro.msfc.nasa.gov/lynx/docs/science/blackholes.html

${ }^{13}$ We report the upper and lower flux limits for a $3 \operatorname{arcsec}$ PSF survey designed as a WFI wedding cake strategy with single tiers of: $4 \times 1 \mathrm{Ms}, 20 \times 300 \mathrm{ks}$, $75 \times 100 \mathrm{ks}$, and $259 \times 10 \mathrm{ks}$, for a total collecting area of $2 \mathrm{~m}^{2}$ at $1 \mathrm{keV}$ and an instrument field of view of $40 \times 40 \mathrm{arcmin}$.
}

only gas accretion. Starting from an initial mass of $10^{5} \mathrm{M}_{\odot}$, the seed experiences Eddington-limited growth during the $250 \mathrm{Myr}$ of 'isolated' evolution of the system (i.e. before a galaxy merger occurs Valiante et al. 2018b). In this case, both the stellar emission and accreting BH intrinsic emission have been reprocessed through the host galaxy ISM, combining the GQD model predictions (galaxy SFR, BH accretion rate, ISM metallicity, dust-to-gas ratio, etc.) with the radiative transfer code CLOUDY (Ferland et al. 2013; see Valiante et al. $2018 \mathrm{~b}$, for details.).

The SED of the growing heavy seed is shown at three different ages (labelled with their emission redshift), ending at $z=10$ when the $\mathrm{BH}$ mass is $\sim 1.5 \times 10^{7} \mathrm{M}_{\odot}$. Although we include the starburst contribution, in this case the emission is completely dominated by the accreting $\mathrm{BH}$ at all redshifts; in other words, the AGN is way more luminous than the host galaxy stellar component and it is potentially detectable by both Athena and NIRcam onboard JWST (Valiante et al. 2018b).

\section{DISCUSSION}

If the growth of seeds is regulated by gas accretion in haloes experiencing multiple mergers, their modelling encompasses a rich and complex variety of physical processes. During galaxy assembly ruled by mergers and gas inflows from the cosmic web, the formation of binary seeds appears highly probable if not inevitable.

In this work, we used the semi-analytical, data-constrained, hierarchical model GQD (Valiante et al. 2014, 2016, 2018a) to track the formation of SMBHs starting from the first stars and first $\mathrm{BH}$ seeds, light and heavy, following the formation of the earliest $\mathrm{BH}$ binaries and their coalescence driven by triple interactions. We assumed that BBHs form within at most few Myr in 100 per cent of major halo-halo mergers and that a triplet forms in 100 per cent of triple/multiple $\mathrm{BH}$ encounters (see Section 3). The sinking timescale of BHs on kpc-to-pc scales is usually set by dynamical friction against background stars and gas. The halo mass ratio, $\mathrm{BH}$ intrinsic masses (customarily in excess of $10^{6} \mathrm{M}_{\odot}$ ), DM profiles, redshiftdependent gas fraction and galaxy morphology, presence of irregular substructures, and even the spatial and mass resolution of simulations all control the formation/failure of a bound system (see e.g. Callegari et al. 2009; Fiacconi et al. 2013; Capelo et al. 2015; Pfister et al. 2017; Tamburello et al. 2017; Tamfal et al. 2018, and references therein). In zoomed-in high redshift $(z \sim 9)$ simulations of dwarf proto-galaxies, dynamical friction against stars is found to be the main process of $\mathrm{BH}$ orbital decay for $\sim 10^{5} \mathrm{M}_{\odot}$ seeds, while erratic dynamics is seen below this mass, implying either rapid decay or $\mathrm{BH}$ wandering/ejection and the presence of multiple $\mathrm{BHs}$ in a galaxy, each inherited from a different merger (Pfister et al. 2019). Interestingly, at high redshifts $(z>6)$ and for $\mathrm{BHs}$ of $\sim 10^{6} \mathrm{M}_{\odot}$, global or bar-induced torques in some cases appear to be more efficient than dynamical friction in promoting $\mathrm{BH}$ binary formation on time-scales comparable to the local Hubble time at those redshifts (Bortolas et al. 2020). Moreover, additional kpc-scale delays can further alter the above picture (see e.g. Barausse et al. 2020) Yet, the process of light seeds binaries formation/merger is unexplored in cosmological simulations, as capturing their dynamics requires extreme high spatial and mass resolution.

In our approach, cosmologically driven $\mathrm{BH}$ mergers are triggered only via triplet formation following the prescriptions of Bonetti et al. (2018a, b) with the sole difference that we approximate the triple interaction as instantaneous, neglecting the triplet lifetime. This relies on the fact that, although the triplet lifetime shows a lognormal distribution with a mean value of $\sim 250 \mathrm{Myr}$, this is mostly due 
to the dynamical friction phase, which we do not model here. ${ }^{14}$ Once the three-body interaction becomes effective, the associated time-scale to resolve the triplet (either a merger or an ejection) is actually much shorter ( $\sim$ few Myr), justifying our assumption of instantaneous interaction. Triplets also have a limited efficiency (at most $\sim 30$ per cent) in triggering $\mathrm{BH}$ mergers and we expect that a large fraction of triple encounters end up with a 'stalled' leftover system (Bonetti et al. 2018a).

Neglecting the physical delays could imply a higher fraction of mergers at earlier times/at lower mass ranges. Depending on the delay time (i.e. time spent by the triplet before coalescence, as computed by Bonetti et al. 2018b), the merger event could be shifted at lower redshifts and, in the meantime, the inner binary could grow in mass via gas accretion on to the two components (thus changing the merger probability). In addition, when the dynamical merger time-scale is longer than our binary lifetime (defined in Section 4.1), we may expect an additional intruder to interact with the triplet, further complicating the scenario (and the description of dynamical processes). We plan to study these more complex aspects in a future work.

In our implementation, we also neglected the effect of stellar hardening and viscous migration in driving the two BHs down to the GW-driven domain. It should be noted that, considering additional hardening time-scales due to binary-gas disc interactions and/or stellar-dominated processes may contribute to the population of merging BHs (e.g. Bortolas et al. 2016; Bortolas, Mapelli \& Spera 2018a; Arca Sedda et al. 2019b; Biava et al. 2019; Souza Lima et al. 2020, and references therein). Therefore, in this respect our results should be viewed as conservative and, in a forthcoming work, we aim at introducing an improved description of more realistic $\mathrm{BH}$ dynamics and merger time-scales to analyse their impact on $\mathrm{SMBH}$ growth and $\mathrm{BH}$ merger history. We expect efficient stellar/gas hardening to have a major impact on the 'stalled' leftover binaries (i.e. in the case in which triple interactions fail in triggering $\mathrm{BH}$ coalescence) and/or when the triplet-driven mergers require long time-scales ( $>1 \mathrm{Gyr}$, as e.g. following the ejection of one $\mathrm{BH}$; Bonetti et al. 2018a). In environments in which stellar/gas-driven shrinking proceeds on relatively short time-scales $(<100-300 \mathrm{Myr})$, the binary may be efficiently driven down to the GW emission phase (Arca Sedda et al. 2019b; Bortolas et al. 2018b) even before a triplet forms, thus affecting the number and redshift of the mergers.

Full control of the $\mathrm{BH}$ dynamics down to the $\mathrm{GW}$-driven domain is fundamental when predicting the rate of $\mathrm{BH}$ coalescences alongside the hierarchical assembly of galaxies. This has been investigated in a number of studies so far, under different assumptions and approaches regarding the merger time-scales (e.g. Enoki et al. 2005; Sesana et al. 2011; Klein et al. 2016; Tamanini et al. 2016; Ricarte \& Natarajan 2018; Bonetti et al. 2019; Dayal et al. 2019; Katz et al. 2020; Volonteri et al. 2020, and references therein).

In our analysis, we simulate the histories of SMBHs and their host galaxy, forming in rare, highly biased regions of the Universe. Thus, a direct comparison of our results with the studies mentioned above is difficult, as these usually describe populations of galaxies/AGNs in an 'average' region of the Universe.

\footnotetext{
${ }^{14}$ It should also be noted that the stellar environment of Bonetti et al. simulations was calibrated against low- $z$ galaxies, and as such are not directly applicable to the problem at hand. First, due to the shorter local dynamical time, one might expect a much faster evolution in dense protogalaxies at high redshift. Secondly, the evolution might well be dominated by dynamical friction against the dense gaseous background rather than stars.
}

Using the SAM Delphi, Dayal et al. (2019) find that binaries with total masses of $10^{3.5}-10^{5} \mathrm{M}_{\odot}$ are detectable, with an $\mathrm{S} / \mathrm{N}>7$, in the redshift range $z \sim 5-13$, with the large fraction being mergers of light seeds (called 'Type 1' mergers). This is consistent with our predictions shown in Fig. 4.

Within a zoomed-in, re-simulated, region of $\left(15 h^{-1} \mathrm{Mpc}\right)^{3}$ extracted from the BlueTides cosmological hydrodynamic simulation, Huang, Feng \& Di Matteo (2019) examined the early growth of $z$ $>6 \mathrm{SMBHs}$, running different sets of simulations for three different BH seed masses: $5 \times 10^{3}, 5 \times 10^{4}$, and $5 \times 10^{5} h^{-1} \mathrm{M}_{\odot}$. All seed scenarios eventually converge to form SMBH of $\sim 10^{9} \mathrm{M}_{\odot}$ provided that the halo mass threshold to $\mathrm{BH}$ seed mass ratio is the same (constant). In their simulations, the rate/number of $\mathrm{BH}$ mergers is higher in the low-mass seed scenario (eight mergers), as lighter seeds are more common/abundant than the more massive ones. Four of such mergers occur at $z>12$ with total masses of $10^{4}-10^{6} \mathrm{M}_{\odot}$, thus being potentially detectable with LISA. This result is consistent with our findings in the $z_{\mathrm{QSO}}=6.4$ merger history. In contrast, as a consequence of the different $\mathrm{BH}$ seeding and dynamics prescriptions, their massive seed model $\left(\sim 5 \times 10^{5} \mathrm{M}_{\odot}\right)$ does not predict any merger until $z<6$.

\section{CONCLUSIONS}

Our model suggests that a statistical inference of the mass distribution and relative occurrence of the earliest BH mergers, if/when provided by the combination of ET and LISA detections, will offer a unique insight into the earliest $\mathrm{BH}$ seed formation epoch and its evolution across cosmic time. On the other hand, thanks to the better sensitivity of $\mathrm{CE}$ at higher frequencies and thus lower $\mathrm{BH}$ stellar masses, $\mathrm{CE}$ observations will be fundamental to study the complementary population of stellar-mass BBHs with masses $\leq 100 \mathrm{M}_{\odot}$ out to $z \sim$ $10-15$.

As commonly expected, an observational signature of the light $\mathrm{BH}$ seed channel could be the higher occupation fraction and thus a higher merger rate compared to the heavy seed one (e.g. Sesana, Volonteri \& Haardt 2007; Sesana et al. 2011; Klein et al. 2016; Ricarte \& Natarajan 2018; Bonetti et al. 2019; Huang et al. 2019). X-rays, deep field, observations may help in discriminating the imprints of different $\mathrm{BH}$ seeds (e.g. in the high- $z$ luminosity functions), although it will be challenging to uniquely disentangle their EM observational features (Pacucci et al. 2015; Natarajan et al. 2017; Volonteri et al. 2017; Ricarte \& Natarajan 2018; Valiante et al. 2018b).

Detecting the GW signals of BHs of $\sim 100 \mathrm{M}_{\odot}$ up to $\sim 10^{7} \mathrm{M}_{\odot}$ from cosmic dawn to the present will enable us to unveil if seeds are the fil rouge connecting the stellar BHs to the SMBHs or if a desert and genetic division exists between the two populations (Colpi 2019).

Fig. 8 summarizes the limiting GW and EM sensitivities in the $m_{\mathrm{BH}, \mathrm{T}}-z$ plane. Waterfall plots for LISA (blue) and ET (red) for an $\mathrm{S} / \mathrm{N}=10$ are reported as a function of the merging $\mathrm{BH}$ binary mass ratio $q=1,0.5,0.3$, and 0.1 while upper limits show the highest redshift at which an accreting $\mathrm{BH}$ of given mass (equivalent to the mass of a $\mathrm{BBH}$ ) is detectable by Athena (orange), at the deepest survey layer limiting flux of $2.4 \times 10^{-17} \mathrm{erg} \mathrm{s}^{-1} \mathrm{~cm}^{-2}$, and by Lynx (black) at the limiting sensitivity level of $10^{-19} \mathrm{erg} \mathrm{s}^{-1} \mathrm{~cm}^{-2}$. These upper limits are computed assuming that accreting BHs emit at the Eddington luminosity, $L=L_{\mathrm{Edd}}$, with 10 per cent of the flux emerging in the hard X-ray bandwidth, suitably redshifted. ${ }^{15}$ The

\footnotetext{
${ }^{15} \mathrm{~A}$ hard-to-soft X-ray luminosity conversion factor of 1.35 is taken into account, for a power-law spectrum with a photon index of $\Gamma=1.9$.
} 


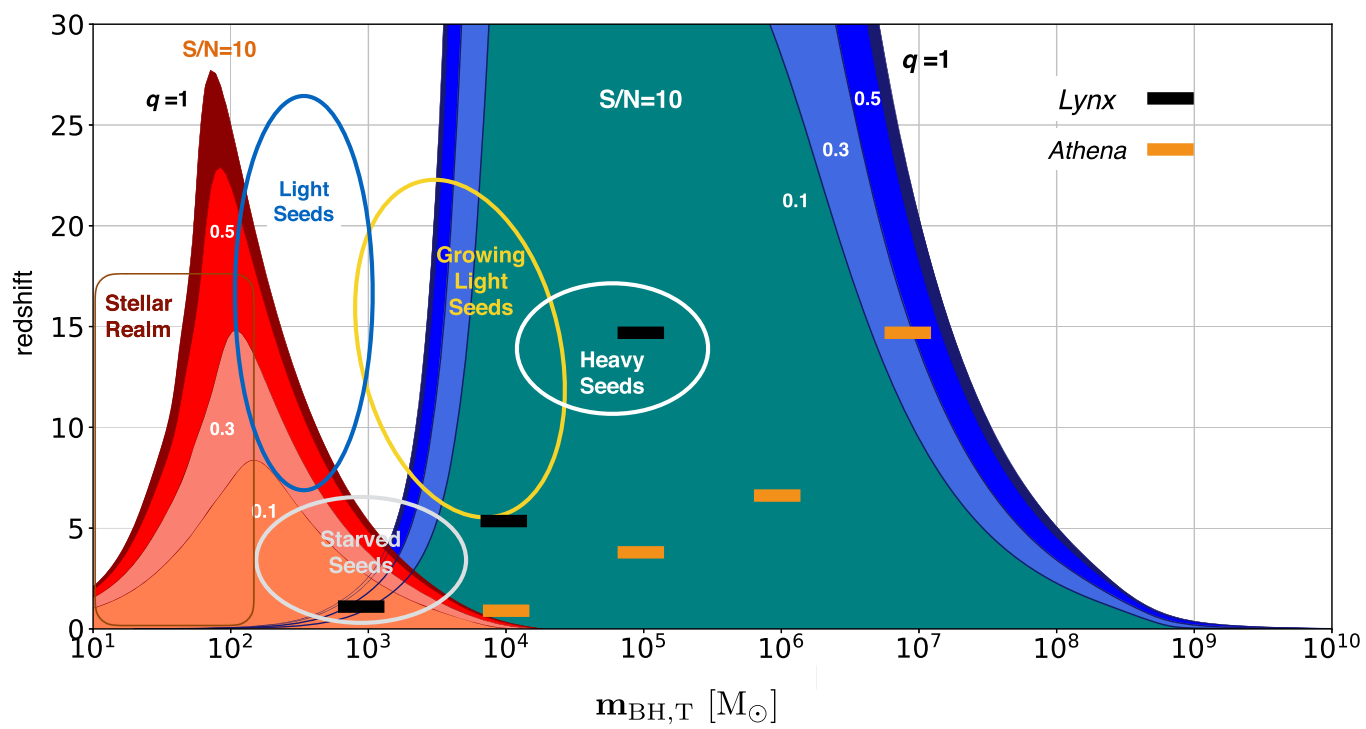

Figure 8. The GW and EM landscape. Colour-coded areas give the average GW horizon computed for a detection threshold equal to $\mathrm{S} / \mathrm{N}=10$ : Contour lines refer to binaries with mass ratios $q=1,0.5,0.3$, and 0.1 in both the ET and LISA bandwidths. Upper limits (shown as thick horizontal bars) indicate the sensitivity of the deepest pointing, in the [0.5-2] keV observed band, by Athena (orange) and Lynx (black) given the limiting fluxes of $2.4 \times 10^{-17}$ and $10^{-19}$ $\mathrm{erg} \mathrm{s}^{-1} \mathrm{~cm}^{-2}$, respectively. The upper limits are inferred assuming that BHs are emitting at the Eddington limit and adopting a bolometric correction $\left(L_{\mathrm{X}} / L_{\mathrm{bol}}\right)$ of 10 per cent. Ellipses highlight the islands in the $z-m_{\mathrm{BH}}$, T plane where light (blue) and heavy (white) seeds are expected to form as well as where light seeds are expected to grow via accretion and mergers (yellow). The transit to the SMBH domain covers the entire LISA area and EM observations are key to discover the high-mass tail of the SMBH distribution. The light-grey ellipse below $z \sim 5$ marks the population of long-living 'starved' seeds. Note that in this island, coordinated multiband observations are possible having LISA the capability to first follow the early inspiral phase in intermediate-mass BHs and ET to catch the subsequent merger phase, enhancing the ability to carry on precise measurements of the source parameters also at $z \sim 5$ (Jani et al. 2019 ). The islands have overlap with the GW horizon, but an empty inaccessible region is present between ET and LISA, corresponding to the deci-Hz GW domain. The island corresponding to the stellar realm is included, on the left, for comparison.

ellipses drawn in the figure mark the different regions where light (blue ellipse) and heavy (white ellipse) seeds are expected to form and where growing (yellow ellipse) and 'starved' (grey ellipse) light seeds are expected to reside. For comparison, on the left of the figure we also plot the region corresponding to stellar-mass BHs, under the assumptions that these come from Pop II stellar binaries formed in the field and in higher metallicity environments, and that their total masses can extend up to few $\sim 100 \mathrm{M}_{\odot}$, with a potential superposition with our 'starved' seed population.

ET with sensitivity down to a few $\mathrm{Hz}$ shall have the unique capability of discovering the earliest $\mathrm{BH}$ binaries in the range of stellar BHs, light and medium-weight seeds forming in the Universe, probing the existence of these rare transitional objects that happen to evolve into SMBHs through gas accretion and mergers under favourable cosmic conditions. ET will be the only instrument that will let us discover light BH seeds forming at cosmic dawn.

On the other hand, if these seeds fail to grow, they may be present in galaxies at lower redshift. $3 \mathrm{G}$ detectors shall have the sensitivity to reveal such failed seeds, which we define as 'starved' seeds. Discovering BHs in this uncharted territory will be groundbreaking.

As light/medium-weight seeds evolve via accretion and mergers, they will transit across the LISA bandwidth and the match between ET and LISA events will statistically shed light on the seeding mechanism. LISA also has the potential to detect the rare heavy seeds in their transit to become supermassive. The lack of events on the right side of ET waterfall plot could be an indication that only heavy seeds are the progenitor of the SMBHs or that light seeds grow at a very fast (super-Eddington) rate, following their formation without experiencing cosmologically driven mergers.
Finally, there are planned experimental programs employing atom interferometers, like the Atom Interferometer Observatory and Network (Badurina et al. 2020), that propose to explore GWs in the mid-frequency range, filling the gap between CE/ET and LISA.

Deep EM observations of galaxies and active BHs at redshifts $z \sim$ 8-10 with forthcoming and next-generation facilities combined with independent observations of coalescing BHs with $\mathrm{GW}$ observatories will offer the first ever view of the young Universe, by capturing the first moment of star and $\mathrm{BH}$ formation in the earliest galaxies. While JWST, Athena, and Lynx (if in operation) will see little patches of the deep Universe to unveil the dawn of galaxies and accreting BHs, ET and LISA will witness the dawn of BH binaries.

In a companion paper, we will investigate in detail the expected accuracy of parameter recovery from GW signals from light seeds observed in CE/ET and from growing light seeds and heavy seeds in LISA. As discussed in Section 4, the waveform in the CE/ET sensitive band will comprise only a few cycles and consequently accurate recovery of parameters will be challenging. We will investigate whether, with accurate waveforms incorporating spin effects and higher harmonics, we will enable differentiation of candidate light seeds from $\mathrm{BH}$ mergers of stellar origin. We will also carry on parameter estimation analysis of the high-redshift seeds detectable with LISA during their slow, adiabatic inspiral.

As discussed in Section 6, including the physics of $\mathrm{BH}$ dynamics (e.g. realistic astrophysical time delays) is critical for any reliable characterization of the merging $\mathrm{BBH}$ populations across the cosmic history (as well as for the evaluation of the merger rates). We aim to improve the model presented here including proper binary formation/merger time-scales and extending our investigations to (i) additional seed flavours (e.g. including the medium-weight channel 
and a mass function for heavy seeds) and to (ii) wider ranges of DM halo masses and redshifts (e.g. to quantify $\mathrm{BBH}$ occupation fraction and LISA/ET/CE merger rates across cosmic epochs).

\section{ACKNOWLEDGEMENTS}

We thank the anonymous referee for the constructive comments, Evan Hall for sharing agreement on the ET waterfall plots, and Bangalore Sathyaprakash for enlightening discussions. RV thanks Luca Zappacosta for productive discussions and suggestions. RV, RS, and MC acknowledge support from the Amaldi Research Center funded by the Ministero dell'Istruzione dell'Università e della Ricerca (MIUR) programme Dipartimento di Eccellenza (CUP: B81I18001170001). MB, MC, FH, AM, and RV acknowledge the networking support by the Cooperation In Science and Technology (COST) Action CA16104 and funding from the Istituto Nazionale di Astrofisica (INFN), "Teoria delle Onde Gravitazionali" (TEONGRAV) specific initiative, and MIUR under the grant Progetti di Ricerca di Rilevante Interesse Nazionale (PRIN) 2017-MB8AEZ. SF and CM acknowledge support from the Science and Technology Facilities Council (STFC) grant ST/L000962/1 and European Research Council Consolidator Grant 647839.

\section{DATA AVAILABILITY}

The simulated data underlying this article will be shared on reasonable request to the corresponding author.

\section{REFERENCES}

Abbott B. P. et al., 2017, Class. Quantum Gravity, 34, 044001

Abel T., Bryan G. L., Norman M. L., 2002, Science, 295, 93

Agarwal B., Khochfar S., Johnson J. L., Neistein E., Dalla Vecchia C., Livio M., 2012, MNRAS, 425, 2854

Aird J. et al., 2013, preprint (arXiv:1306.2325)

Alexander T., Natarajan P., 2014, Science, 345, 1330

Amaro-Seoane P. et al., 2017, preprint (arXiv:1702.00786)

Arca Sedda M. et al., 2019a, preprint (arXiv:1908.11375)

Arca Sedda M., Berczik P., Capuzzo-Dolcetta R., Fragione G., Sobolenko M., Spurzem R., 2019b, MNRAS, 484, 520

Askar A., Szkudlarek M., Gondek-Rosińska D., Giersz M., Bulik T., 2017, MNRAS, 464, L36

Badurina L. et al., 2020, J. Cosmol. Astropart. Phys., 2020, 011

Baldassare V. F., Reines A. E., Gallo E., Greene J. E., 2015, ApJ, 809, L14

Bañados E. et al., 2016, ApJS, 227, 11

Bañados E. et al., 2018, Nature, 553, 473

Barausse E., 2012, MNRAS, 423, 2533

Barausse E., Morozova V., Rezzolla L., 2012, ApJ, 758, 63

Barausse E., Dvorkin I., Tremmel M., Volonteri M., Bonetti M., 2020, preprint (arXiv:2006.03065)

Begelman M. C., Blandford R. D., Rees M. J., 1980, Nature, 287, 307

Begelman M. C., Volonteri M., Rees M. J., 2006, MNRAS, 370, 289

Biava N., Colpi M., Capelo P. R., Bonetti M., Volonteri M., Tamfal T., Mayer L., Sesana A., 2019, MNRAS, 487, 4985

Bischetti M. et al., 2019, A\&A, 628, A118

Bonetti M., Haardt F., Sesana A., Barausse E., 2016, MNRAS, 461, 4419

Bonetti M., Sesana A., Barausse E., Haardt F., 2018a, MNRAS, 477, 2599

Bonetti M., Haardt F., Sesana A., Barausse E., 2018b, MNRAS, 477, 3910

Bonetti M., Sesana A., Haardt F., Barausse E., Colpi M., 2019, MNRAS, 486, 4044

Booth C. M., Schaye J., 2009, MNRAS, 398, 53

Bortolas E., Gualandris A., Dotti M., Spera M., Mapelli M., 2016, MNRAS, 461, 1023

Bortolas E., Mapelli M., Spera M., 2018a, MNRAS, 474, 1054
Bortolas E., Gualandris A., Dotti M., Read J. I., 2018b, MNRAS, 477, 2310

Bortolas E., Capelo P. R., Zana T., Mayer L., Bonetti M., Dotti M., Davies M. B., Madau P., 2020, MNRAS, 498, 3601

Brightman M. et al., 2013, MNRAS, 433, 2485

Bromm V., Loeb A., 2004, New Astron., 9, 353

Callegari S., Mayer L., Kazantzidis S., Colpi M., Governato F., Quinn T., Wadsley J., 2009, ApJ, 696, L89

Capelo P. R., Volonteri M., Dotti M., Bellovary J. M., Mayer L., Governato F., 2015, MNRAS, 447, 2123

Chon S., Omukai K., 2020, MNRAS, 494, 2851

Chon S., Hirano S., Hosokawa T., Yoshida N., 2016, ApJ, 832, 134

Colpi M., 2014, Space Sci. Rev., 183, 189

Colpi M., 2019, in Latif M., Schleicher D., eds, Formation of the First Black Holes, World Scientific Publishing Co. Pte. Ltd., Singapore, p. 241

Colpi M. et al., 2019, preprint (arXiv:1903.06867)

Cowie L. L., Barger A. J., Bauer F. E., González-López J., 2020, ApJ, 891, 69

Dal Canton T., Mangiagli A., Noble S. C., Schnittman J., Ptak A., Klein A., Sesana A., Camp J., 2019, ApJ, 886, 146

Davies M. B., Miller M. C., Bellovary J. M., 2011, ApJ, 740, L42

Dayal P., Rossi E. M., Shiralilou B., Piana O., Choudhury T. R., Volonteri M., 2019, MNRAS, 486, 2336

de Bennassuti M., Schneider R., Valiante R., Salvadori S., 2014, MNRAS, 445, 3039

de Bennassuti M., Salvadori S., Schneider R., Valiante R., Omukai K., 2017, MNRAS, 465, 926

Devecchi B., Volonteri M., Rossi E. M., Colpi M., Portegies Zwart S., 2012, MNRAS, 421, 1465

Di Carlo U. N. et al., 2020, MNRAS, 498, 495

Di Matteo T., Springel V., Hernquist L., 2005, Nature, 433, 604

Dijkstra M., Ferrara A., Mesinger A., 2014, MNRAS, 442, 2036

Dominik M., Belczynski K., Fryer C., Holz D. E., Berti E., Bulik T., Mandel I., O’Shaughnessy R., 2012, ApJ, 759, 52

Dominik M., Belczynski K., Fryer C., Holz D. E., Berti E., Bulik T., Mandel I., O'Shaughnessy R., 2013, ApJ, 779, 72

Dominik M. et al., 2015, ApJ, 806, 263

Dosopoulou F., Antonini F., 2017, ApJ, 840, 31

Enoki M., Inoue K. T., Nagashima M., Sugiyama N., 2005, Annu. Rep. Natl. Astron. Obs. Japan, 7, 34

Fan X. et al., 2001, AJ, 122, 2833

Fan X., Hennawi J. F., Richards G. T., Strauss M. A., Schneider D. P., Donley J. L., Young J. E., Annis e. a., 2004, AJ, 128, 515

Ferland G. J. et al., 2013, Rev. Mex. Astron. Astrofis., 49, 137

Fiacconi D., Mayer L., Roškar R., Colpi M., 2013, ApJ, 777, L14

Graziani L., Schneider R., Marassi S., Del Pozzo W., Mapelli M., Giacobbo N., 2020, MNRAS, 495, L81

Habouzit M., Volonteri M., Latif M., Dubois Y., Peirani S., 2016, MNRAS 463,529

Hall E. D., Evans M., 2019, Class. Quantum Gravity, 36, 225002

Heger A., Woosley S. E., 2002, ApJ, 567, 532

Heger A., Woosley S. E., 2010, ApJ, 724, 341

Heger A., Fryer C. L., Woosley S. E., Langer N., Hartmann D. H., 2003, ApJ, 591,288

Hild S. et al., 2011, Class. Quantum Gravity, 28, 094013

Hirano S., Hosokawa T., Yoshida N., Umeda H., Omukai K., Chiaki G., Yorke H. W., 2014, ApJ, 781, 60

Hirano S., Hosokawa T., Yoshida N., Omukai K., Yorke H. W., 2015 MNRAS, 448, 568

Hirano S., Yoshida N., Sakurai Y., Fujii M. S., 2018, ApJ, 855, 17

Huang K.-W., Feng Y., Di Matteo T., 2019, MNRAS, 496, 1

Inayoshi K., Omukai K., 2012, MNRAS, 422, 2539

Inayoshi K., Omukai K., Tasker E., 2014, MNRAS, 445, L109

Inayoshi K., Hirai R., Kinugawa T., Hotokezaka K., 2017, MNRAS, 468, 5020

Inayoshi K., Visbal E., Haiman Z., 2020, Annual Review of Astronomy and Astrophysics, 58, 27

Jani K., Shoemaker D., Cutler C., 2019, Nat. Astron., 4, 260

Johnson J. L., Haardt F., 2016, PASA, 33, e007 
Kalogera V. et al., 2019, BAAS, 51, 242

Katz M. L., Kelley L. Z., Dosopoulou F., Berry S., Blecha L., Larson S. L., 2020, MNRAS, 491, 2301

Kelley L. Z., Blecha L., Hernquist L., 2017, MNRAS, 464, 3131

Khan F. M., Fiacconi D., Mayer L., Berczik P., Just A., 2016, ApJ, 828, 73

Klein A. et al., 2016, Phys. Rev. D, 93, 024003

Kormendy J., Ho L. C., 2013, ARA\&A, 51, 511

Kozai Y., 1962, AJ, 67, 591

Larson R. B., 1998, MNRAS, 301, 569

Latif M. A., Ferrara A., 2016, PASA, 33, e051

Latif M. A., Schleicher D. R. G., Schmidt W., Niemeyer J. C., 2013, MNRAS, 436, 2989

Lidov M. L., 1962, Planet. Space Sci., 9, 719

Luo J. et al., 2016, Class. Quantum Gravity, 33, 035010

Lupi A., Colpi M., Devecchi B., Galanti G., Volonteri M., 2014, MNRAS, 442,3616

McGee S., Sesana A., Vecchio A., 2020, Nat. Astron., 4, 26

Madau P., Rees M. J., 2001, ApJ, 551, L27

Madau P., Haardt F., Dotti M., 2014, ApJ, 784, L38

Maggiore M. et al., 2020, JCAP, 03, 050

Mapelli M., 2016, MNRAS, 459, 3432

Mapelli M., Giacobbo N., 2018, MNRAS, 479, 4391

Mapelli M., Giacobbo N., Ripamonti E., Spera M., 2017, MNRAS, 472, 2422

Mapelli M., Giacobbo N., Santoliquido F., Artale M. C., 2019, MNRAS, 487, 2

Marassi S., Graziani L., Ginolfi M., Schneider R., Mapelli M., Spera M., Alparone M., 2019, MNRAS, 484, 3219

Marconi A., Risaliti G., Gilli R., Hunt L. K., Maiolino R., Salvati M., 2004, MNRAS, 351, 169

Matsuoka Y. et al., 2018, ApJ, 869, 150

Mayer L., Kazantzidis S., Madau P., Colpi M., Quinn T., Wadsley J., 2007, Science, 316, 1874

Mayer L., Fiacconi D., Bonoli S., Quinn T., Roškar R., Shen S., Wadsley J., 2015, ApJ, 810, 51

Merloni A., Rudnick G., Di Matteo T., 2004, MNRAS, 354, L37

Mezcua M., Civano F., Fabbiano G., Miyaji T., Marchesi S., 2016, ApJ, 817, 20

Mezcua M., Civano F., Marchesi S., Suh H., Fabbiano G., Volonteri M., 2018, MNRAS, 478, 2576

Nardini E. et al., 2015, Science, 347, 860

Natarajan P., Pacucci F., Ferrara A., Agarwal B., Ricarte A., Zackrisson E., Cappelluti N., 2017, ApJ, 838, 117

Pacucci F., Ferrara A., Volonteri M., Dubus G., 2015, MNRAS, 454, 3771

Pezzulli E., Volonteri M., Schneider R., Valiante R., 2017a, MNRAS, 466, 2131

Pezzulli E., Volonteri M., Schneider R., Valiante R., 2017b, MNRAS, 471, 589

Pfister H., Lupi A., Capelo P. R., Volonteri M., Bellovary J. M., Dotti M., 2017, MNRAS, 471, 3646

Pfister H., Volonteri M., Dubois Y., Dotti M., Colpi M., 2019, MNRAS, 486, 101

Press W. H., Schechter P., 1974, ApJ, 187, 425

Punturo M. et al., 2010, Class. Quantum Gravity, 27, 194002

Regan J. A., Visbal E., Wise J. H., Haiman Z., Johansson P. H., Bryan G. L., 2017, Nat. Astron., 1, 0075

Reines A. E., Volonteri M., 2015, ApJ, 813, 82

Reinoso B., Schleicher D. R. G., Fellhauer M., Klessen R. S., Boekholt T. C. N., 2018, A\&A, 614, A14

Reitze D. et al., 2019, BAAS, 51, 35
Ricarte A., Natarajan P., 2018, MNRAS, 481, 3278

Robson T., Cornish N. J., Liu C., 2019, Class. Quantum Gravity, 36, 105011

Rodriguez C. L., Chatterjee S., Rasio F. A., 2016, Phys. Rev. D, 93, 084029

Ruan W.-H., Guo Z.-K., Cai R.-G., Zhang Y.-Z., 2018, preprint (arXiv:1807 .09495)

Santamaría L. et al., 2010, Phys. Rev. D, 82, 064016

Santoliquido F., Mapelli M., Bouffanais Y., Giacobbo N., Di Carlo U. N., Rastello S., Artale M. C., Ballone A., 2020, ApJ, 898, 152

Sathyaprakash B. et al., 2012, Class. Quantum Gravity, 29, 124013

Sato S. et al., 2017, J. Phys. Conf. Ser., 840, 012010

Schleicher D. R. G., Palla F., Ferrara A., Galli D., Latif M., 2013, A\&A, 558, A59

Schneider R., Ferrara A., Natarajan P., Omukai K., 2002, ApJ, 571, 30

Schneider R., Ferrara A., Salvaterra R., Omukai K., Bromm V., 2003, Nature, 422, 869

Schneider R., Omukai K., Bianchi S., Valiante R., 2012, MNRAS, 419, 1566

Schneider R., Graziani L., Marassi S., Spera M., Mapelli M., Alparone M., Bennassuti M. d., 2017, MNRAS, 471, L105

Schulze A. et al., 2019, MNRAS, 488, 1180

Sesana A., Volonteri M., Haardt F., 2007, MNRAS, 377, 1711

Sesana A., Gair J., Berti E., Volonteri M., 2011, Phys. Rev. D, 83, 044036

Shen Y. et al., 2011, ApJS, 194, 45

Souza Lima R., Mayer L., Capelo P. R., Bortolas E., Quinn T. R., 2020, ApJ, 899,126

Sugimura K., Matsumoto T., Hosokawa T., Hirano S., Omukai K., 2020, ApJ, 892, L14

Tamanini N., Caprini C., Barausse E., Sesana A., Klein A., Petiteau A., 2016, J. Cosmol. Astropart. Phys., 2016, 002

Tamburello V., Capelo P. R., Mayer L., Bellovary J. M., Wadsley J. W., 2017, MNRAS, 464, 2952

Tamfal T., Capelo P. R., Kazantzidis S., Mayer L., Potter D., Stadel J., Widrow L. M., 2018, ApJ, 864, L19

Trakhtenbrot B., 2020, preprint (arXiv:2002.00972)

Umeda H., Hosokawa T., Omukai K., Yoshida N., 2016, ApJ, 830, L34

Valiante R., Schneider R., Salvadori S., Bianchi S., 2011, MNRAS, 416, 1916

Valiante R., Schneider R., Salvadori S., Gallerani S., 2014, MNRAS, 444, 2442

Valiante R., Schneider R., Volonteri M., Omukai K., 2016, MNRAS, 457, 3356

Valiante R., Agarwal B., Habouzit M., Pezzulli E., 2017, PASA, 34, e031

Valiante R., Schneider R., Graziani L., Zappacosta L., 2018a, MNRAS, 474, 3825

Valiante R., Schneider R., Zappacosta L., Graziani L., Pezzulli E., Volonteri M., 2018b, MNRAS, 476, 407

Van Wassenhove S., Capelo P. R., Volonteri M., Dotti M., Bellovary J. M., Mayer L., Governato F., 2014, MNRAS, 439, 474

Volonteri M., 2010, A\&AR, 18, 279

Volonteri M., Haardt F., Madau P., 2003, ApJ, 582, 559

Volonteri M., Silk J., Dubus G., 2015, ApJ, 804, 148

Volonteri M., Reines A. E., Atek H., Stark D. P., Trebitsch M., 2017, ApJ, 849,155

Volonteri M. et al., 2020, MNRAS, 498, 2219

Wise J. H., Regan J. A., O'Shea B. W., Norman M. L., Downes T. P., Xu H., 2019, Nature, 566, 85

Yang J. et al., 2020, ApJ, 897, L14

Yoshida N., Omukai K., Hernquist L., 2008, Science, 321, 669

This paper has been typeset from a $\mathrm{T}_{\mathrm{E}} \mathrm{X} / \mathrm{L} \mathrm{T} \mathrm{E} \mathrm{X}$ file prepared by the author. 